\title{
AUFSATZ
}

\section{Innerfraktionelle Geschlossenheit im Spiegel der Politikwissenschaft. Stand der Forschung und notwendige Weiterführungen}

\author{
Erik Fritzsche
}

Wenn in Parlamenten knappe Abstimmungen zu kontroversen politischen Inhalten anstehen, ist dies überall auf der Welt ein Thema für jene, die - wie meist die Medien - nicht nur an allen sachlichen Belangen effektiven Regierens interessiert sind, sondern eben auch am zuspitzenden, bisweilen dramatischen Charakter von Politik Gefallen finden. Es fragen sich interessierte Beobachter, das politische Geschehen vermittelnde Berichterstatter und Politikwissenschaftler gleichermaßen: Was verursacht die Geschlossenheit von Parlamentsfraktionen? Was veranlasst frei gewählte Abgeordnete, mit einer politischen Gruppe zu stimmen? Welche Bedingungen führen zur sichtbarsten Manifestation des durch Gruppen geprägten politischen Wettbewerbs? Und woran scheitert dies manchmal?

Das politikwissenschaftliche Interesse an diesem Phänomen geht allerdings über diesen - ohnehin nur gelegentlich vorhandenen - Nachrichtenwert weit hinaus. Für das parlamentarische Regierungssystem ist unbestritten, dass die Geschlossenheit eine Grundvoraussetzung für sein Funktionieren darstellt: Sowohl die Stabilität der Regierung als auch die klare Trennung der regierenden von der opponierenden Rolle ist vom Vorliegen weitgehend geschlossener Fraktionen abhängig. Im präsidentiellen Regierungssystem sind die Fraktionen dagegen von der Bürde stabilen Regierens weitgehend entlastet. Doch hier haben unterschiedliche Geschlossenheitsniveaus durchaus ganz verschiedene Konsequenzen: Durch geschlossene Parlamentsfraktionen können einerseits in Phasen des divided government Systemblockaden hervorgerufen werden. Andererseits können allzu wenig geschlossen agierende Parlamentsfraktionen die Transaktionskosten der Mehrheitsfindung erhöhen und politische Verantwortlichkeiten verwischen. Zudem kann die horizontale Gewaltenteilung (checks and balances) durch extrem geschlossene und gegebenenfalls vom Präsidenten kontrollierte Parteien leicht ausgehebelt werden. Es ist daher gar nicht einfach, das wünschenswerte Geschlossenheitsniveau für das präsidentielle System anzugeben ${ }^{1}$, nicht zuletzt hin-

1 Vgl. Scott Mainwaring / Matthew S. Shugart, Conclusion: Presidentialism and the Party System, in: dies. (Hrsg.), Presidentialism and Democracy in Latin America, Cambridge 1997, S. 418 - 421. Nicht minder relevant sind Panorama und Ursachen innerfraktioneller Geschlossenheit für theoriestrategische Zwecke: Zum einen sind immer dann, wenn relativ geschlossen agierende Kollektivakteure angenommen werden (so etwa gängig bei der Erklärung von Zustandekommen, Zerbrechen und Arbeiten von Koalitionen), Theorien zu den Ursachen solcher Geschlossenheit Teile des Erklärenden. Außerdem sind Anwendungsvoraussetzungen, etwa jene der Vetospielertheorie, durch einen Blick auf die Geschlossenheit zu prüfen. Zu letzterem siehe Steffen Kailitz, Ein Unterschied wie Tag und Nacht? Fraktionsgeschlossenheit in Parlamentarismus und Präsidentialismus, in: ZPol, 18. Jg. (2008), H. 3, S. $291-324$. 
sichtlich der Stabilität dieses Regierungssystemtyps. ${ }^{2}$ Infolgedessen bedarf das Phänomen der Fraktionsgeschlossenheit der Erhellung. Auch mit Blick auf ihre geringe Varianz im parlamentarischen Regierungssystem und der dortigen Bedeutung einheitlichen Abstimmens wird die Kausalanalyse des Phänomens nicht irrelevant. Schließlich garantiert nichts, dass sich Fraktionsgeschlossenheit auch einstellen wird, wenn es systemisch wichtig erscheint. Und mehr noch: Selbst wenn hohe Geschlossenheit tatsächlich beobachtet wird, müssen die einschlägigen Ursachen noch lange nicht wünschenswert sein.

Die folgende Auseinandersetzung mit dem internationalen Schrifttum wird über die angebotenen forschungsleitenden Modelle und gängigen Forschungshypothesen ebenso berichten wie über das empirische Material, wie es in vielen Studien präsentiert wird. ${ }^{3}$ Auf dieser Grundlage können Defizite der Forschungslage benannt und einige Vorschläge für die künftige Forschung unterbreitet werden.

\section{Forschungsleitende Modelle im Überblick}

Mitunter wird zwar kritisiert, dass nützliche forschungsleitende und speziell vergleichsanleitende Modelle fehlten ${ }^{4}$, was allerdings an den wenigen vergleichenden Studien liegt, die zur Fraktionsgeschlossenheit überhaupt vorliegen. Doch es gibt durchaus nennenswerte Bemühungen, erkenntnisleitende Vergleichskategorien zu systematisieren. Den locus classicus der - insbesondere vergleichenden - Forschung hat Ergun Ozbudun geschaffen. ${ }^{5}$ Seine Studie stellt vor allem einen systematisch-theoriegeleiteten Überblick der bis Ende der 1960er Jahre verfassten Arbeiten dar. Was die Darstellung und den Test verschiedener theoretischer Ansätze angeht, hat Thomas Saalfeld schon Mitte der 1990er Jahre mit seiner Monographie zur Geschlossenheit der Fraktionen des Deutschen Bundestages hinreichend Systematisierung betrieben, um auch vergleichende Forschung anzuleiten. ${ }^{6}$ Modelle mit sehr abstrakten

2 Beispielsweise wird geringe Geschlossenheit in Verbindung mit hoher Fragmentierung des Parteiensystems und ausgeprägten präsidentiellen Machtbefugnissen für problematisch gehalten (so Scott Mainwaring I Matthew S. Shugart, Juan Linz, Presidentialism and Democracy. A Critical Appraisal, in: Comparative Politics, 29. Jg. (1997), H. 4, S. 449 - 471). Dagegen wird die interne Geschlossenheit von Vetospielern, wenn sie in großer Zahl auftreten, für stabilitätsgefährdend erachtet (so George Tsebelis, Veto Players, New York 2002).

3 Der Verfasser hat sich bemüht, auch jene Beiträge einzubeziehen, die nicht in einer von ihm gesprochenen Sprache erschienen sind. Vorwiegend wird neuere Literatur berichtet. Zur (spärlichen) Literaturlage vor 1970 siehe Ergun Ozbudun, Party Cohesion in Western Democracies: A Causal Analysis, Beverly Hills 1970; ein - hier nicht zu leistender - Bericht einschlägiger ideengeschichtlicher Quellen wählte als fruchtbaren Startpunkt wohl Edmund Burkes berühmte Rede an die Wähler in Bristol und zöge weiter bis zu den klassischen Parteienforschern der ersten Hälfte des 20. Jahrhunderts (Robert Michels, Moisei Ostrogorski, Elmer E. Schattschneider).

4 Vgl. statt vieler John E. Owens, Explaining Party Cohesion and Discipline in Democratic Legislatures: Purposiveness and Contexts, in: Journal of Legislative Studies, 9. Jg. (2004), H. 4, S. 12 40, S. 15.

5 Siehe Ergun Ozbudun, a.a.O. (Fn. 3).

6 Siehe Thomas Saalfeld, Parteisoldaten und Rebellen. Eine Untersuchung zur Geschlossenheit der Fraktionen im Deutschen Bundestag (1949 - 1990), Opladen 1995. Dass dessen Klarheit im Arbeiten mit den einschlägigen Theorien nicht weiter rezipiert wurde, liegt wohl auch daran, dass der theoriebildende und -testende Vergleich in der deutschen Parlamentarismusforschung ohnehin nicht allzu populär ist (vgl. Werner J. Patzelt, Parlamentarismus, in: Ludger Helms / Uwe Jun 
Begriffen, die sich deshalb für weitgespannte Vergleiche gut eignen7, legten Werner J. Patzelt und Torbjörn Bergman u.a. vor. ${ }^{8}$ Beide Modelle wurden wiederum von Romy Messerschmidt aufgegriffen, die in ihrer Analyse der Entstehung und Bedeutung innerfraktioneller Geschlossenheit in der Assemblée Nationale der V. Republik Frankreichs alle einschlägigen Wirkfaktoren erfasste und in einem Kausalmodell zusammenführte. ${ }^{9}$ Ferner legte Reuven Y. Hazan eine nützliche Unterscheidung von Faktoren vor: Einerseits solche, die eine - soziologisch zu erklärende - Übereinstimmung von Präferenzen, Normen, Perzeptionen und Weltbildern von Fraktionsmitgliedern („Kohäsion“) bewirken, andererseits - rationalwahlanalytisch argumentierend - jene Strukturen und Prozesse, die individuelle Kalküle von Abgeordneten prägen und so auf deren Verhalten wirken („Disziplin“). ${ }^{10}$ Ein sehr umfassendes und zugleich recht konkretes Modell verfasste David M. Olson. ${ }^{11}$ Besonders betont wurden sozialisatorische und politisch-kulturelle Faktoren von Asbjørn Skjeveland sowie von Torben K. Jensen. ${ }^{12}$ Weitere Modelle präsentierten Ulrich Sieberer sowie John M. Carey: Beide betrieben Vergleichsanalysen mittels statistischer Modelle bei mittleren Fallzahlen. ${ }^{13}$ Bei Shaun Bowler, David M. Farrell und Richard S. Katz sowie bei Thomas Saalfeld sind „Inventare" von plausiblen Kausalfaktoren zu finden. ${ }^{14}$

(Hrsg.), Politische Theorie und Regierungslehre. Eine Einführung in die politikwissenschaftliche Institutionenforschung, Frankfurt am Main 2004, S. 97 - 129).

7 Zum Umgang mit möglichen Abstraktionsniveaus in der Vergleichsforschung siehe Giovanni Sartori, Concept Misformation in Comparative Politics, in: American Political Science Review, 64. Jg. (1970), H. 4, S. 1033 - 1053.

8 Siehe Werner J. Patzelt, Party Cohesion and Party Discipline in German Parliaments, in: Journal of Legislative Studies, 9. Jg. (2003), H. 4, S. 102 - 115; Torbjörn Bergman / Wolfgang C. Müller / Kaare Strom / Magnus Blomgren, Democratic Delegation and Accountability: Cross-National Patterns, in: Kaare Strøm / Wolfgang C. Müller / Torbjörn Bergman (Hrsg.), Delegation and Accountability in Parliamentary Democracies, Oxford 2003, S. 109 - 220.

9 Siehe Romy Messerschmidt, Fraktionenparlament Nationalversammlung. Entstehung und Bedeutung innerfraktioneller Geschlossenheit, Wiesbaden 2005. Eine ähnlich gute Aufbereitung möglicher Kategorien, wie sie bei Thomas Saalfeld, a.a.O. (Fn. 6), oder Romy Messerschmidt geleistet wurde, findet sich üblicherweise in Einzelfallstudien, insbesondere bei Wolfgang C. Müller / Marcelo Jenny / Barbara Steininger, Die österreichischen Abgeordneten, Wien 2001 und bei Laura Valencia Escamilla, La Disciplina Parlamentaria en México. LVII Legislatura de la Cámera de Diputados, Mexico D. F. 2005.

10 Siehe Reuven Y. Hazan, Does Cohesion Equal Discipline? Towards a Conceptual Delineation, in: Journal of Legislative Studies, 9. Jg. (2003), H. 4, S. 1 - 11.

11 Siehe David M. Olson, Cohesion and Discipline Revisited: Contingent Unity in the Parliamentary Party Group, in: Journal of Legislative Studies, 9. Jg. (2003), H. 4, S. $164-178$.

12 Siehe Asbjørn Skjaveland, A Danish Party Cohesion Cycle, in: Scandinavian Political Studies, 22. Jg. (1999), H. 2, S. 121 - 136; Torben K. Jensen, Party Cohesion, in: Peter Esaiasson / Knut Heidar (Hrsg.), Beyond Westminster and Congress. The Nordic Experience, Columbus 2000, S. $210-236$.

13 Siehe John M. Carey, Competing Principals, Political Institutions, and Party Unity in Legislative Voting, in: American Journal of Political Science, 51. Jg. (2007), H. 1, S. 92 - 107; Ulrich Sieberer, Party Unity in Parliamentary Democracies. A Comparative Analysis, in: Journal of Legislative Studies, 12. Jg. (2006), H. 2, S. $150-178$.

14 Siehe Shaun Bowler / David M. Farrell / Richard S. Katz, Party Cohesion, Party Discipline, and Parliaments, in: dies. (Hrsg.), Party Discipline and Parliamentary Government, Columbus 1999, S. 3 - 22; Thomas Saalfeld, Determinanten der Fraktionsdisziplin: Deutschland im internationalen Vergleich, in: Steffen Ganghof / Philip Manow (Hrsg.), Mechanismen der Politik. Strategische Interaktionen im deutschen Regierungssystem, Frankfurt am Main 2005, S. 35 - 71. Eine Tren- 


\section{Arbeiten zu zentralen Hypothesen:Welche Bedingungen können den Grad der Fraktionsgeschlossenheit erklären?}

Für den gegenwärtigen Stand der Theoriebildung kann auf Grundlage der genannten Beiträge folgende Feststellung getroffen werden: Allen Modellen ist die Vorstellung zumindest nicht völlig fremd, dass Geschlossenheit aufgrund gemeinsam geteilter Bewertungen von politisch-inhaltlichen Alternativen zustande kommt, mithin eine gemeinsame Handlungsgrundlage der Fraktionsmitglieder besteht. Diesen theoretischen Vorstellungsinhalt etikettiert man am besten mit „Fraktionskohäsion“. Daher ist in der Forschung die Denkfigur mehr oder minder üblich, nach der sozialisationstheoretische und politisch-kulturelle Variablen eine beträchtliche Wirkung entfalten, beispielsweise durch die soziale Homogenität der Fraktionsmitglieder oder deren Sozialisations-, Rekrutierungs- und Karrieremuster sowie durch das von diesen Größen beförderte Potenzial eines Mit- und Füreinanders. Auf einem durchaus schon erheblichen Niveau innerfraktioneller Geschlossenheit stellt man sich das Zustandekommen von größtmöglicher Geschlossenheit, gewissermaßen in einem zweiten Schritt, durch die Effekte angekündigter beziehungsweise vorweggenommener Sanktionen und Anreize vor. Diese können von den Parteien, Fraktionen sowie sonstigen Akteuren aus dem Umfeld der parlamentarischen Gruppen ausgehen. Dies lässt sich treffend mit „Fraktionsdisziplin“ bezeichnen. Zumindest implizit wird bei entsprechenden Forschungshypothesen davon ausgegangen, dass die spezifischen Strukturen des Parlamentarismus die Kosten-Nutzen-Kalküle der Abgeordneten prägen und auf diese Weise ihr Verhalten beeinflussen. Systemtheoretische Überlegungen werden dergestalt mit einer zunächst einfachen - rationalwahlanalytischen Handlungstheorie verbunden. Fraktionskohäsion und Fraktionsdisziplin tragen beide, allerdings aufgrund eben verschiedener Ursachen, zu jenem Phänomen bei, das als einheitliches Abstimmen im Parlament beobachtet wird und mit „innerfraktioneller Geschlossenheit“ oder „Fraktionsgeschlossenheit" am besten bezeichnet ist. ${ }^{15}$

Die genannten Modelle unterscheiden außerdem solche Ursachen, die vom Inneren des Parlaments her wirken und von dort aus die Kalküle der Akteure prägen. Hierzu gehört ganz wichtig - das Verhältnis zur Regierung und damit insbesondere die Frage nach dem Regierungssystemtyp. Hierhin gehören auch die Struktur des Ausschusssystems und - damit verbunden - die parlamentarische Arbeitsteilung. Oft wurden in diese Kategorie auch solche Einflussgrößen eingeordnet, die sich innerhalb der Fraktionen befinden: vor allem das Sanktionspotential der Fraktionsführerschaft, die finanzielle, personelle, prozedurale Verteilung machtrelevanter Ressourcen zwischen den Abgeordneten und der Fraktionsführung, die Führungspraxen und politische Patronage.

nung von Erklärungen, die auf Anreizen im Inneren des Parlaments beruhen („one-arena models") und solchen, die überdies aufgrund des (Wieder-)Wahlzusammenhangs bestehen (sich daher außerhalb des Parlaments befinden: „two-arena models“) findet sich bei Shaun Bowler, Parties in Legislatures: Two Competing Explanations, in: Russell J. Dalton / Martin P. Wattenberg (Hrsg.), Parties without Partisans: Political Change in Advanced Industrial Democracies, Oxford 2000, S. 129 - 153 (mit weiteren Nachweisen).

15 Die konsequente Durchsetzung dieses Sprachgebrauches wäre zu wünschen und ist durch theoretische Überlegungen auch plausibel begründet, siehe Reuven Y. Hazan, a.a.O. (Fn: 10); Romy Messerschmidt, a.a.O. (Fn. 9); und jüngst Janina Thiem, Nationale Parteien im Europäischen Parlament - Delegation, Kontrolle und politischer Einfluss, Wiesbaden 2009, S. 125 ff. 
Neben diesen innerparlamentarischen Faktoren wurden Faktoren modelliert, die von außerhalb des Parlaments - nicht notwendigerweise jedoch außerhalb der Parteien - wirken, zum Beispiel die Wahlsysteme und die Kandidatenselektionsprozesse sowie die Kampagnenfähigkeit der jeweiligen Akteure. Außerdem fanden die föderalen Strukturelemente und der Einfluss von Regierungen auf die Entscheidungen „ihrer“ Partei(en) (speziell die Kandidatenauslese) Beachtung. Ferner wurden Parteiensystemeigenschaften - insbesondere Polarisierung und Fragmentierung - in die Modelle integriert und die Stellung der konkret untersuchten Partei im Parteienwettbewerb als Einflussgröße betrachtet (Regierungs- versus Oppositionsfraktion, Größe der Fraktion, Knappheit der Mehrheiten, politisch-ideologische Stellung der Fraktion). Auch der Einfluss einer spezifischen „Fraktionskultur“ wurde thematisiert. ${ }^{16}$

Werner J. Patzelt integrierte die Erwartungen der Bürger, vor allem ihre hohe Wertschätzung des innerparteilichen Konsenses, als wirkmächtige Größe ausdrücklich in ein Pfeilmodell und versuchte, sie systematisch empirisch zu erfassen. ${ }^{17}$ Der Wert des politischen „Markennamens“ einer Partei wurde nur selten zur Erklärung herangezogen, wohl hauptsächlich wegen fehlender sich zum Vergleich eignender Daten. Ähnliche Probleme behindern auch den Test jener Hypothese, die ein geringeres Geschlossenheitsniveau erwarten lässt, wenn Abgeordnete die Möglichkeit besitzen, Wählerstimmen aufgrund ihrer persönlichen Verhaltensweisen auf sich zu ziehen (personal vote). Bei derzeitiger Datenlage wird dergleichen immerhin indirekt erfassbar, indem von Wahlsystemeigenschaften auf entsprechende Anreize zur Kultivierung eines solchen personenbezogenen Wahlverhaltens geschlossen wird. ${ }^{18}$

Es lassen sich zudem eine Reihe von Studien finden, die einzelne Variablen theoretisch ausarbeiten beziehungsweise deren Effekte empirisch zu erfassen versuchen. Zur Agendasetzung hat Herbert Döring Analysen vorgelegt, die die auf diesem Gebiet maßgeblichen Bei-

16 Im Übrigen werden mitunter, insbesondere bei Romy Messerschmidt, a.a.O. (Fn. 9), (und auch bei Werner J. Patzelt, a.a.O. [Fn. 8]), explizit Überlegungen über die Kovarianz verschiedener unabhängiger Variablen (!) angestellt.

17 Siehe Werner J. Patzelt, a.a.O. (Fn. 8); ders., Wider das Gerede vom ,Fraktionszwang? Funktionslogische Zusammenhänge, populäre Vermutungen und die Sicht der Abgeordneten, in: ZParl, 29. Jg. (1998), H. 2, S. 323 - 347, S. 330. Vgl. jedoch das Sozialisation besonders hervorhebende Modell im Beitrag von Asbjørn Skjaveland, a.a.O. (Fn. 12). Siehe ebenso Ergun Ozbuduns Ausführungen zu den liberalen, radikalen und kollektivistischen Repräsentationstheorien, a.a.O. (Fn. 3), S. 363 - 378; vgl. auch die Überlegungen der Motivlagen zur „Parteiloyalität" bei Torben K. Jensen, a.a.O. (Fn. 12). An dieser Stelle wären systematische Betrachtungen von Repräsentation als wissenschaftliches und populäres Konzept einzugliedern (siehe hierzu Hanna F. Pitkin, The Concept of Representation, Berkeley 1967 sowie ihre Rezeption und Weiterführung in: Werner J. Patzelt, Abgeordnete und Repräsentation. Amtsverständnis und Wahlkreisarbeit, Passau 1993. Zur Wirkung solcher Vorstellungen auf die Seltenheit namentlicher Abstimmungen im Nationalrat der Schweiz infolge einer tief in der Parlamentstradition verwurzelten liberalen Repräsentationsvorstellung siehe Prisca Lanfranchi / Ruth Lüthi, Cohesion of Party Groups and Interparty Conflicts in the Swiss Parliament: Roll Call Voting in the National Council, in: Shaun Bowler 1 David M. Farrell / Richard S. Katz (Hrsg.), a.a.O. (Fn. 14), S. 99 - 120.

18 Siehe John M. Carey / Matthew Soberg Shugart, Incentives to Cultivate a Personal Vote: a Rank Ordering of Electoral Formulas, in: Electoral Studies, 14. Jg. (1995), H. 4, S. 417 - 439; Paul Mitchell, Voters and their Representatives: Electoral Institutions and Delegation in Parliamentary Democracies, in: European Journal of Political Research, 37. Jg. (2000), H. 3, S. 335 - 351. 
träge von Gary W. Cox und Mathew D. McCubbins ${ }^{19}$ sowie John D. Huber reflektierten. ${ }^{20}$ Die Anpassung innerparlamentarischer Regeln zur Stabilisierung und Organisation von präsidententreuen Mehrheiten in der russischen Staatsduma ist unter anderem Thema einer Arbeit von Thomas F. Remington. ${ }^{21}$ Speziell zum Verhältnis von Regierung und Mehrheitsfraktion(en) ist der sich durch formale Theoriebildung auszeichnende Aufsatz von Daniel Diermeier und Timothy J. Feddersen zu nennen. ${ }^{22}$ Der systematischste Test des Einflusses von Regierungssystemen wurde von Steffen Kailitz verfasst. ${ }^{23}$ Bjørn Erik Rasch untersuchte die Wirkung verschiedener Weisen, aufeinander folgende Abstimmungsvorlagen zu präsentieren. ${ }^{24}$ Die Interaktion von Wahlsystemen und Prozessen der Kandidatenselektion hat Alejandro Poiré analysiert. ${ }^{25}$ Effekte des Wahlsystems werden, da so viele Störvariablen kontrolliert werden können, gern an Mischwahlsystemen erforscht. ${ }^{26}$ Mit den

19 Siehe Gary W. Cox / Mathew D. McCubbins, Legislative Leviathan. Party Government in the House, Berkeley 1993; dies., Setting the Agenda. Responsible Party Government in the U.S. House of Representatives, Cambridge 2005.

20 Siehe insbesondere Herbert Döring, Party Discipline and Government Imposition of Restrictive Rules, in: Journal of Legislative Studies, 9. Jg. (2003), H. 4, S. 147 - 163; ders., Controversy, Time Constraint, and Restrictive Rules, in: Herbert Döring / Mark Hallerberg (Hrsg.), Patterns of Parliamentary Behavior: Passage of Legislation across Western Europe, Aldershot 2004, S. 141 168; ders., Worauf gründet sich die Agenda-Setzer-Macht der Regierung?, in: Steffen Ganghof I Philip Manow (Hrsg.), a.a.O. (Fn. 14), S. 109 - 148; John D. Huber, Rationalizing Parliament. Legislative Institutions and Party Politics in France, Cambridge 1996.

21 Siehe Thomas F. Remington, Presidential Support in the Russian State Duma, in: Legislative Studies Quarterly, 31. Jg. (2006), H. 1, S. 5 - 30.

22 Siehe Daniel Diermeier / Timothy J. Feddersen, Cohesion in Legislatures and the Vote of Confidence Procedure, in: American Political Science Review, 92. Jg. (1998), H. 3, S. 611 - 621; John D. Huber, a.a.O. (Fn. 20).

23 Siehe Steffen Kailitz, a.a.O. (Fn. 1), der auch Effekte von föderalen Systemelementen und Wahlsystemen testet.

24 Speziell mit Bezug zur Geschlossenheit der Fraktionen siehe Bjørn Erik Rasch, Electoral Systems, Parliamentary Committees, and Party Discipline: The Norwegian Storting in a Comparative Perspective, in: Shaun Bowler / David M. Farrell / Richard S. Katz (Hrsg.), a.a.O. (Fn. 14), S. 121 140; zur Theorie siehe zudem - mit weiteren Verweisen - Bjørn Erik Rasch, Parliamentary Floor Voting Procedures and Agenda Setting in Europe, in: Gerhard Loewenberg / Peverill Squire / Roderick D. Kiewiet (Hrsg.), Legislatures. Comparative Perspectives on Representative Assemblies, Ann Arbor 2002, S. 269 - 287.

25 Siehe Alejandro Poiré, Do Electoral Institutions Affect Party Discipline?, or: Nominations Rule! Comparative Evidence on the Impact of Nomination Procedures on Party Discipline, ITAM Departmento Académico de Ciencia Política, Working Papers on Political Science 2002-03, México D.F. 2003.

26 Zum EP siehe Thorsten Faas, To Defect or not to Defect? National, Institutional and Party Group Pressures on MEPs and their Consequences for Party Group Cohesion in the European Parliament, in: European Journal of Political Research, 42. Jg. (2003), H. 5, S. 841 - 866; Simon Hix, Electoral Institutions and Legislative Behavior: Explaining Voting Defection in the European Parliament, in: World Politics, 56. Jg. (2004), H. 2, S. 194 - 223; zur Lage in Deutschland siehe Michael Becher / Ulrich Sieberer, Discipline, Electoral Rules and Defection in the Bundestag, 1983-94, in: German Politics, 17. Jg. (2008), H. 3, S. 293 - 304; zu Mexiko siehe Laura Valencia Escamilla, a.a.O. (Fn. 9), sowie Jeffrey A. Weldon, Factores Institucionales y Políticos de la Disciplina Partidaria en la Cámera de Diputados de México, 1998 - 2002. Instituto Tecnológico Autónomo de México, México D.F. 2002 (verfügbar unter http://usmex.ucsd.edu/research/conf_ pdfs/weldon.pdf, Abruf am 20. Oktober 2008); zu Russland siehe Moshe Haspel / Thomas F. Remington / Steven S. Smith, Electoral Institutions and Party Cohesion in the Russian Duma, in: 
Möglichkeiten der Disziplinierung von Regierungsparteien durch vertragsähnliche Arrangements zwischen Fraktion und Abgeordnetem sowie deren Absicherung durch "Screening“, Selektion, „Monitoring“, Hierarchie und Einbindung von Vetospielern beschäftigte sich Sam Depauw. ${ }^{27}$ Asbjørn Skjaveland befasste sich - inspiriert von der US-amerikanischen Diskussion - mit der Wirkung von nahenden Wahlen auf die Fraktionsgeschlossenheit in Dänemark. ${ }^{28}$ Thomas Saalfeld gab einen Überblick über die Implikationen von Zweikammersystemen. ${ }^{29}$ John M. Carey versuchte, dem Einfluss von Wiederwahlverboten - unter anderem - auf die Fraktionsgeschlossenheit in Costa Rica, Venezuela und den Parlamenten der Gliedstaaten der USA nachzugehen. Er ging dabei auch der Frage nach, welche Anreizäquivalente zum parlamentarischen Karriereinteresse auf diese Abgeordneten wirken könnten. ${ }^{30}$ Eine (eher beiläufige) Rolle spielte die Fraktionsgeschlossenheit in einigen Sammelbänden, die sich mit Fraktionen und ihrem Kontext in einer Reihe von Ländern sehr detailliert beschäftigten. ${ }^{31}$

\section{Das verfügbare empirische Material: Analysen zur Ausprägung und zu den Ursachen der Fraktionsgeschlossenheit}

Welche Arten von Studien zu konkreten Parlamenten und ihren Fraktionen vorliegen, lässt sich wie folgt zusammenfassen: Es dominieren Einzelfalluntersuchungen, die nur mitunter in komparativer Perspektive verfasst sind und sich häufig bei (mindestens) einem der oben

Journal of Politics, 60. Jg. (1998), H. 2, S. 417 - 439; Steven S. Smith / Thomas F. Remington, The Politics of Institutional Choice: the Formation of the Russian State Duma, Princeton 2001; Thomas F. Remington / Jana Kunicová, Mandates, Parties and Dissent: Effect of Electoral Rules on Parliamentary Party Cohesion in the Russian State Duma, 1994-2003, in: Party Politics, 14. Jg. (2008), H. 5, S. 555 - 574; zu Italien nach 1993 siehe Federico Ferrara, Frogs, Mice and Mixed Electoral Institutions: Party Discipline in Italy's XIV Chamber of Deputies, in: Journal of Legislative Studies, 10. Jg. (2004), H. 4, S. 10 - 31; zu Litauen siehe (mit einigen weiteren Nachweisen theoretischer Literatur zu den verhaltensprägenden Effekten von Mischwahlsystemen) Terry D. Clark / Žilvinas Martinaitis / Ramünas Dilba, Electoral Mandate and Party Cohesion: Does It Matter in Lithuania?, in: Journal of Communist Studies and Transition Politics, 24. Jg. (2008), H. 3, S. 317 - 337. Siehe ferner den Befund des Vergleiches der Fraktionen in Ungarn, Russland und der Ukraine bei Frank C. Thames, Party Strength and the Mandate Divide in Hungary, Russia, and Ukraine, in: Comparative Political Studies, 38. Jg. (2005), H. 3, S. 282 - 303.

27 Siehe Sam Depauw, Government Party Discipline in Parliamentary Democracies: The Cases of Belgium, France and the United Kingdom in the 1990s, in: Journal of Legislative Studies, 9. Jg. (2003), H. 4, S. $130-146$.

28 Siehe Asbjørn Skjaveland, a.a.O (Fn. 12).

29 Siehe Thomas Saalfeld, a.a.O. (Fn. 14), mit weiteren Literaturhinweisen; vgl. auch die Darstellung zu den eingeschränkten Möglichkeiten, als Abgeordneter im deutschen Föderalismus zu wirken, bei Werner J. Patzelt, What Can an Individual MP Do in German Parliamentary Politics?, in: Journal of Legislative Studies, 5. Jg. (1999), H. 3, S. 23 - 52.

30 Siehe John M. Carey, Term Limits and Legislative Representation, Cambridge 1996.

31 Siehe Knut Heidar / Ruud Koole (Hrsg.), Parliamentary Party Groups in European Democracies. Political Parties behind Closed Doors, London 2000, darin insbesondere ihre einleitenden theoretischen Überlegungen: Approaches to the Study of Parliamentary Party Groups, S. 4 - 22. Ferner siehe Ludger Helms (Hrsg.), Parteien und Fraktionen. Ein internationaler Vergleich, Opladen 1999; Lawrence D. Longley / Reuven Y. Hazan (Hrsg.), The Uneasy Relationship between Parliamentary Members and Leaders, London 2000. 
angeführten forschungsleitenden Modelle bedienen. Systematisch-theorieorientierte beziehungsweise theoriegenerierende und -testende Vergleiche existieren daneben nur sehr wenig, sogar mit nur mittleren Fallzahlen sind derartige Forschungsdesigns selten.

\subsection{Vergleichende Studien}

Noch überwiegend induktiv vorgehend stellt die Arbeit von Ergun Ozbudun eigentlich kaum mehr als einen Forschungsüberblick dar - dabei jedoch damals wie heute äußerst instruktiv für insbesondere den qualitativen Vergleich. ${ }^{32}$ Am ehesten in jene Tradition ließe sich die lateinamerikanische Fraktionen in den Blick nehmende Analyse von Scott Morgenstern einordnen, die allerdings in methodischer Hinsicht um einiges weiter fortgeschritten ist. ${ }^{33}$ Lobenswert ist besonders, dass diese Studie zusammenwachsen lässt, was auch zusammengehört: narrativ-gestalterschließende Betrachtungen von Einzelfällen (dabei dennoch klar durch die verwendeten Vergleichskategorien geprägt) mit den vielen Innovationen der quantitativen Analyse von namentlichen Abstimmungen, wie sie von der USamerikanischen Parlamentarismusforschung entwickelt wurden. ${ }^{34}$ Scott Morgensterns Analyse befindet sich daher in einer sehr nutzbringenden und sowohl dem Fallmaterial als auch dem Forschungsstand angemessenen Mittellage zwischen Fall- und Theorieorientierung.

Eher erstere bieten zwei Vergleiche ähnlicher Systeme derselben Region. Scott Mainwaring und Matthew S. Shugart trugen systematisch zusammen, was sie aus einer Reihe von Länderstudien lernen konnten. ${ }^{35}$ Torben K. Jensen ging den Mustern innerfraktioneller Geschlossenheit im Rahmen einer umfassenden Analyse nordeuropäischer Parlamente auf den Grund. ${ }^{36}$ Eher kursorisch bei der Abhandlung des Verhältnisses von Partei und Fraktion wurde Fraktionsgeschlossenheit auch in einigen Sammelbänden betrachtet. ${ }^{37}$

Theorieorientierter und -testender Vergleich mit möglichst vielen Fällen findet man bei Ulrich Sieberer, der sich westeuropäischen Parlamenten widmete. ${ }^{38}$ Ähnlich, jedoch ein deutlich heterogeneres Fallmaterial betrachtend, ist die Studie von John M. Carey angelegt, der im Übrigen in seiner neuesten Arbeit lateinamerikanische, US-amerikanische und europäische Parlamente betrachtet. ${ }^{39} \mathrm{Ob}$ Kandidatenselektion oder Wahlsysteme den prägenderen Einfluss haben, hat Alejandro Poiré zu beantworten versucht. ${ }^{40}$ Mit dem bislang umfänglichsten Datensatz führte Steffen Kailitz eine Vergleichsanalyse durch. ${ }^{41}$

32 Siehe Ergun Ozbudun, a.a.O. (Fn. 3).

33 Siehe Scott Morgenstern, Patterns of Legislative Politics. Roll-Call Voting in Latin America and the United States, Cambridge 2004.

34 Vgl. Keith T. Poole, Spatial models of parliamentary voting, Cambridge 2005.

35 Siehe Scott Mainwaring / Matthew S. Shugart, Conclusion: Presidentialism and the Party System, a.a.O. (Fn. 1).

36 Siehe Torben K. Jensen, a.a.O. (Fn. 12).

37 Siehe Knut Heidar / Ruud Koole (Hrsg.), a.a.O. (Fn. 31); Ludger Helms (Hrsg.), a.a.O. (Fn. 31).

38 Siehe Ulrich Sieberer, a.a.O. (Fn. 13).

39 Siehe John M. Carey, a.a.O. (Fn. 13); ders., Legislative Voting and Accountability, Cambridge 2009.

40 Siehe Alejandro Poiré, a.a.O. (Fn. 25). Dort wird sich auf den Datensatz gestützt von: Kenneth Janda, Political Parties: A Cross-National Survey, New York 1980, insbesondere S. $118-125$.

41 Siehe Steffen Kailitz, a.a.O. (Fn. 1). 
Vergleiche mit (ganz) geringen Fallzahlen, interessiert an der Wirkung von wenigen Variablen stellen folgende Studien dar: Sam Depauw analysierte die Disziplin der Regierungsparteien in Belgien, Frankreich und Großbritannien ${ }^{42}$; Herbert Döring beschäftigte sich mit der Wirkung von Instrumenten der Agendasetzung auf die Geschlossenheit von Fraktionen $^{43}$; John M. Carey versuchte, auf der Basis von Interviewdaten zu ergründen, welchen Responsivitätserfordernissen konfligierender Prinzipale (Partei, Fraktion, Wähler) Abgeordnete in Lateinamerika ausgesetzt sind ${ }^{44}$; Louise K. Davidson-Schmich machte in ihrer innerdeutsche Fälle betrachtenden und innovativen Vergleichsanalyse Hypothesen plausibel, die erwarten lassen, dass es eine zunehmende Angleichung des Abstimmungsverhaltens von Abgeordneten einer Fraktion gäbe, indem Normen internalisiert, zweckmäßige Handlungsmuster erlernt und durch gegebene institutionelle Anreizstrukturen belohnt würden. ${ }^{45}$ Frank C. Thames untersuchte Effekte auf die Geschlossenheit in Ungarn, der Ukraine und Russland. ${ }^{46}$

\subsection{Einzelfallbezogene Studien und Daten zur Fraktionsgeschlossenheit}

Neben diesen Vergleichen hat die Forschung eine ganze Reihe von Einzelfallstudien hervorgebracht, die neben ihren an sich schon verdienstvollen Darstellungen auch für anzustrebende Vergleiche nützliche Daten und hilfreiche Einsichten enthalten.

Parlamente in den USA, in Westeuropa, Skandinavien und das Europäische Parlament (EP) Eine ausgeprägte Diskussion der Geschlossenheit parlamentarischer Parteien gibt es in den USA. Werte der Geschlossenheit samt deren internationaler Einordnung finden sich bei John M. Carey und Scott Morgenstern sowie - für die Zeit vor 1990 - bei Samuel C. Patterson und Gregory A. Caldeira. ${ }^{47}$ Dort wird festgestellt (beliebtester Forschungsgegenstand ist das House of Representatives), dass das Niveau der Geschlossenheit auch im internationalen Vergleich recht gering ist (obwohl die oft anzutreffende Lehrbuchweisheit, es gäbe keine Fraktionsgeschlossenheit im US-Kongress, nicht den Tatsachen entspricht). Daher bewegte die Forscher zweierlei: (1) Wie ist die vorhandene Geschlossenheit ohne die oft den kontinentaleuropäischen Parteien zugeschriebenen Disziplinierungsinstrumente (etwa der Selektion der Kandidaten) zu erklären, und welche Ursachen hat der zu beobachtende Wandel der Fraktionsgeschlossenheit? (2) Welche (macht-)politischen Folgen hat die Geschlossenheit für die betreffenden Parteien und das politische System? Dazu wurden (teils

42 Siehe Sam Depauw, a.a.O. (Fn. 27).

43 Siehe Herbert Döring, a.a.O. (Fn. 20).

44 Siehe John M. Carey, Discipline, Accountability, and Legislative Voting in Latin America, in: Comparative Politics, 35. Jg. (2003), H. 2, S. $191-211$.

45 Siehe Louise K. Davidson-Schmich, The Development of Party Discipline in New Parliaments: Eastern German State Legislatures 1990 - 2000, in: Journal of Legislative Studies, 9. Jg. (2003), H. 4, S. 88 - 101; dies., The Origins of Party Discipline: Evidence from Eastern Germany, in: German Politics and Society, 24. Jg. (2006), H. 2, S. 23 - 43.

46 Siehe Frank C. Thames, a.a.O. (Fn. 26); ders., Discipline and Party Institutionalization in PostSoviet Legislatures, in: Party Politics, 13. Jg. (2007), H. 4, S. $456-477$.

47 Siehe John M. Carey, a.a.O. (Fn. 13); Scott Morgenstern, a.a.O. (Fn. 33); Samuel C. Patterson I Gregory A. Caldeira, Abstimmungskohäsion (Party-Voting) im amerikanischen Kongreß, in: ZParl, 17. Jg. (1986), H. 2, S. $200-223$. 
widerstreitende) Theorien und zum Test der einschlägigen Hypothesen sehr ausgereifte quantitative Methoden entwickelt. ${ }^{48}$

Für Deutschland legte Thomas Saalfeld eine detaillierte und alle relevanten Hypothesen des Forschungsgebietes testende Auswertung aller namentlichen Abstimmungen des Deutschen Bundestages bis 1990 vor. Louise K. Davidson-Schmich lieferte zudem Daten für die Fraktionen der Landtage nach 1990. ${ }^{49}$ Auf Basis von Abgeordneten- und Bürgerbefragungen beschrieb Werner J. Patzelt zwölf zur Geschlossenheit führende "Regelkreisläufe".50 Dessen Ergebnisse sind zudem in Form zweier, das ganze Ursachengefüge darstellender Aufsätze veröffentlicht ${ }^{51}$. Dort wie bei Suzanne S. Schüttemeyer wurden die zentralen Systemzusammenhänge dargelegt. ${ }^{52}$ Ferner untersuchten Michael Becher und Ulrich Sieberer das hier interessierende Phänomen. ${ }^{53}$ Auch einige historische Parlamente und ihre Fraktionen sind erschlossen. ${ }^{54}$ Auf der Grundlage der genannten Studien ist ein sehr detailliertes

48 Siehe als Einstieg in die US-amerikanische Theoriediskussion Steven S. Smith, Positive Theories of Congressional Parties, in: Gerhard Loewenberg / Peverill Squire / Roderick D. Kiewiet (Hrsg.), a.a.O. (Fn. 24), S. 224 - 244; zur Nutzbarkeit dieser Theorien zu Vergleichszwecken siehe Roderick D. Kiewiet / Gerhard Loewenberg / Peverill Squire, The Implications of the Study of the U.S. Congress for Comparative Legislative Research, ebenda, S. 3 - 22. Einen guten Eindruck der methodischen Möglichkeiten liefert Keith T. Poole, a.a.O. (Fn. 34), sowie auch der folgende, hier für die US-amerikanische Parlamentarismusforschung exemplarisch stehende Aufsatz: Nolan McCarty / Keith T. Poole / Howard Rosenthal, The Hunt for Party Discipline in Congress, in: American Political Science Review, 95. Jg. (2001), H. 3, S. 673 - 687. Es kann nur bedauert werden, welche hohen Anforderungen viele dieser analytischen Möglichkeiten an die zugrunde zu legenden Daten stellen. Solches Datenmaterial wird sich selbst in gut dokumentierten Parlamenten selten auffinden lassen. Zur Anwendung der in den USA üblichen Theoreme und Methoden bei einem internationalen Vergleich siehe Scott Morgenstern, a.a.O. (Fn. 33).

49 Siehe Thomas Saalfeld, a.a.O. (Fn. 6), der zudem alle seiner Studie vorangehenden Arbeiten der Forschungen zum Bundestag diskutiert (die hier aus Platzgründen nicht berücksichtigt werden). Siehe Louise K. Davidson-Schmich, The Development of Party Discipline, a.a.O. (Fn. 45); dies., The Origins of Party Discipline, a.a.O. (Fn. 45). Im Übrigen muss hier auf den Bericht des sich erheblich ausweitenden verfassungsrechtlichen, publizistischen und über Fraktionsgeschlossenheit allgemein räsonierenden Schrifttums in Deutschland verzichtet werden (zum Einstieg siehe jedoch mit weiteren Nachweisen: Eberhard Schuett-Wetschky, Grundtypen parlamentarischer Demokratie. Klassisch-altliberaler Typ und Gruppentyp. Unter besonderer Berücksichtigung der Kritik am „Fraktionszwang“, Freiburg (Breisgau) 1984; Werner J. Patzelt, a.a.O. (Fn. 17); Josef Isensee, Fraktionsdisziplin und Amtsgewissen: Verfassungsrechtliche Garantie der Freiheit des Mandats im politischen Prozess, in: Werner J. Patzelt / Martin Sebaldt / Uwe Kranenpohl (Hrsg.), Res publica semper reformanda, Wiesbaden 2007, S. 254 - 267. Außerdem siehe sowohl zur oft falsch wiedergegebenen - Stellung des Parlamentarischen Rates zur Fraktionsgeschlossenheit als auch zur dortigen Praxis Sebastian Galka / Eberhard Schuett-Wetschky, Parlamentarismuskritik und Grundgesetz. Hat der Parlamentarische Rat Fraktionsdisziplin abgelehnt?, in: ZPol, 17. Jg. (2007), H. 4, S. 1095 - 1119).

50 Siehe Werner J. Patzelt, a.a.O. (Fn. 17).

51 Siehe Werner J. Patzelt, Parlamentarische Rekrutierung und Sozialisation: normative Erwägungen, empirische Befunde und praktische Empfehlungen, in: Zeitschrift für Politik, 46. Jg. (1999), H. 3, S. 243 - 281 (mit vielen weiteren Literaturhinweisen); ders., a.a.O. (Fn. 8).

52 Siehe Suzanne S. Schüttemeyer, Fraktionen im Deutschen Bundestag 1949 - 1997. Empirische Befunde und theoretische Folgerungen, Opladen 1998.

53 Siehe Michael Becher / Ulrich Sieberer, a.a.O. (Fn. 26). Sie verwenden die Erklärungen zu den Abstimmungen als Indikator für die Treue der Abgeordneten zur Fraktionslinie.

54 Siehe zum Reichstag in der Weimarer Republik: Heinz Markmann, Das Abstimmungsverhalten der Parteifraktionen, Meisenheim am Glan 1955; Emil Obermann, Alter und Konstanz von Frak- 
Verständnis der Herstellung innerfraktioneller Geschlossenheit erlangt worden, das in Westeuropa als außergewöhnlich gelten kann. ${ }^{55}$

Für das House of Commons des Vereinigten Königreiches ist der Zeitraum nach dem Zweiten Weltkrieg bis 1979 von Philip Norton erschlossen worden. ${ }^{56}$ Daneben gibt es spezielle Studien zur Parlamentsfraktion der Konservativen ${ }^{57}$ und zur Labour Party ${ }^{58}$ sowie allgemein zum Verhältnis von Fraktionsführung und Abgeordneten. ${ }^{59}$ Zudem gab Philip Cowley einen Sammelband heraus, der das Abstimmungsverhalten bei „Gewissensabstimmungen “ in den Blick nimmt. ${ }^{60}$ Für das House of Lords spürte Philip Norton den Mustern dortiger Fraktionsgeschlossenheit nach. ${ }^{61}$

Die französische Assemblée Nationale ist seit der Arbeit von Romy Messerschmidt vor allem für die V. Republik (es finden sich dort jedoch auch Daten zur IV. Republik) aufgear-

tionen: Veränderungen in deutschen Parlamentsfraktionen seit dem Jahre 1920, Meisenheim am Glan 1956. Zur Frankfurter Nationalversammlung siehe Heinrich Best, Die Männer von Bildung und Besitz: Struktur und Handeln parlamentarischer Führungsgruppen in Deutschland und Frankreich 1848/49, Düsseldorf 1990. Zur Fraktionsbindung nach 1819 siehe Helmut Kramer, Fraktionsbindungen in den deutschen Volksvertretungen 1819 - 1849, Berlin 1968.

55 Dies wird auch anschaulich durch eine Fülle weiterer Studien, die sich an den Problemkreis der Fraktionsgeschlossenheit anschließen: zu den Abgeordneten siehe Werner J. Patzelt, Abgeordnete und Repräsentation: Amtsverständnis und Wahlkreisarbeit, a.a.O. (Fn. 17); ders, Deutschlands Abgeordnete: Profil eines Berufsstandes, der weit besser ist als sein Ruf, in: ZParl, 27. Jg. (1996), H. 3, S. 462 - 502; zu den Fraktionen siehe insbesondere Suzanne S. Schüttemeyer, a.a.O. (Fn. 52); zur parlamentarischen und innerfraktionellen Arbeitsteilung siehe dies. sowie Jürgen von Oertzen, Das Expertenparlament. Abgeordnetenrollen in den Fachstrukturen bundesdeutscher Parlamente, Baden-Baden 2006; zum Verhältnis von Parlament und Regierung siehe beispielsweise Manfred Schwarzmeier, Parlamentarische Mitsteuerung. Strukturen und Prozesse informalen Einflusses im Deutschen Bundestag, Wiesbaden 2001.

56 Siehe Philip Norton, Dissension in the House of Commons: Intra-party Dissent in the House of Commons Division Lobbies, 1945 - 1974, London 1975; ders., Dissension in the House of Commons, 1974/79, London 1980.

57 Siehe zur EU-Politik David Baker / Andrew Gamble / Steve Ludlam / David Seawright, Backbenchers with Attitude: A Seismic Study of the Conservative Party and Dissent on Europe, in: Shaun Bowler / David M. Farrell / Richard S. Katz (Hrsg.), a.a.O. (Fn. 14), S. 72 - 98; zur Legislatur von 1992 Philip Cowley / Philip Norton, Rebels and Rebellions: Conservative MPs in the 1992 Parliament, in: British Journal of Politics and International Relations, 1. Jg. (1999), H. 1, S. 84 - 105, und zwischen 1979 und 1988 Thomas Saalfeld, Mrs Thatcher's Poodle? Zum Verhältnis zwischen Regierung und Konservativer Fraktion, 1979 - 1988, in: Roland Sturm (Hrsg.), Thatcherismus. Eine Bilanz nach zehn Jahren, Bochum 1990, S. 17 - 48. Den zunehmend von lokalen Seiten ausgehenden Druck auf die Abgeordneten zeigen auf: Paul F. Whitley / Patrick Seyd, Discipline in the British Conservative Party: The Attitudes of Party Activists toward the Role of Their Members of Parliament, in: Shaun Bowler / David M. Farrell / Richard Katz (Hrsg.), a.a.O. (Fn. 14), S. $53-71$.

58 Siehe für 1974 bis 1979 Brian J. Gaines / Geoffrey Garrett, The Calculus of Dissent: Party Discipline in the British Labour Government, in: Political Behavior, 15. Jg. (1993), H. 2, S. 113 135; für 1997 bis 2001 Philip Cowley, Revolts and Rebellions: Parliamentary Voting under Blair, London 2002; ders. / Mark Stuart, In Place of Strife? The PLP in Government, 1997 - 2001, in: Political Studies, 51. Jg. (2003), H. 2, S. $315-331$.

59 Siehe Philip Norton, The Individual Member in the British House of Commons: Facing both Ways and Marching Forward, in: Journal of Legislative Studies, 5. Jg. (1999), H. 3, S. 53 - 74.

60 Siehe Philip Cowley (Hrsg.), Conscience and Parliament, London 1998.

61 Siehe Philip Norton, Cohesion without Discipline: Party Voting in the House of Lords, in: Journal of Legislative Studies, 9. Jg. (2003), H. 4, S. $57-72$. 
beitet. ${ }^{62}$ Verhältnismäßig gut ist auch die Forschungslage zur Abgeordnetenkammer in Belgien. Sam Depauw analysierte den Zeitraum von 1991 bis 1995 und wertete dabei Interviewdaten zur innerfraktionellen Sanktionslogik aus. ${ }^{63}$

Da vor 1993 geheime Abstimmungen in Italiens Parlament Standard waren, gibt es keine Studien zu Ergebnissen namentlicher Abstimmungen. Nichtsdestoweniger zeigen einige Schlagworte der um Italiens Parlamentarismus kreisenden Diskussion an - zum Beispiel ,parlamentarischer Individualismus' und ,franchi tiratori'64 („Heckenschützen“) -, dass auch dort die Frage nach den Ursachen der Fraktionsgeschlossenheit beziehungsweise ihres Fehlens einige Bedeutung besitzt. ${ }^{65}$ Aufschlussreich in dieser Hinsicht ist der Beitrag von Luca Verzichelli und Maurizio Cotta, die unter anderem zeigten, wie Koalitionen aufgrund innerfraktioneller Konflikte zerbrechen. ${ }^{66}$ Als klassisches Diktum zu den Leistungen italienischer Regierungen kann das von Giuseppe DiPalma konstatierte Dilemma vom „Regieren ohne Überleben" gelten. ${ }^{67}$ Ferner geben die Beiträge von David Hine und Maurizio Cotta einen ersten Überblick über die Labilität parlamentarischer Mehrheiten und das schwierige Regieren in Italiens Erster Republik. ${ }^{68}$ Für Italien nach 1993 untersuchten Massimiliano Landi und Riccardo Pelizzo erstmals Daten namentlicher Abstimmungen. ${ }^{69}$ Auch James L.

62 Dabei lassen ihr theoretisches Modell, die geführten Interviews mit Abgeordneten und die Auswertung aller relevanten Sekundärliteratur kaum Wünsche offen, Romy Messerschmidt, a.a.O. (Fn. 9); siehe auch Frank L. Wilson / Richard Wiste, Party Cohesion in the French National Assembly: 1958 - 1973, in: Legislative Studies Quarterly, 1. Jg. (1976), H. 4, S. 467 - 490. Zur IV. Republik siehe Duncan MacRae Jr., Parliament, Parties, and Society in France: 1946-1958, New York 1967.

63 Siehe Sam Depauw, Parliamentary Party Cohesion and the Scarcity of Sanctions in the Belgian Chamber of Representatives (1991 - 1995), in: Res Publica, 41. Jg. (1999), H. 1, S. 15 - 39. Abgerundet wird das Verständnis der Prozesse durch Lieven de Winter / Patrick Dumont, PPGs in Belgium: Subjects of Partitocratic Dominion, in: Knut Heidar / Ruud Koole (Hrsg.), a.a.O. (Fn. 31), S. $106-129$.

64 Vgl. Maurizio Cotta, The Rise and Fall of the „Centrality” of the Italian Parliament: Transformations of the Executive-Legislative Subsystem after the Second World War, in: Gary W. Copeland I Samuel C. Patterson (Hrsg.), Parliaments in the Modern World. Changing Institutions, Ann Arbor 1994, S. $59-84$.

65 Zur Einführung mit vielen weiteren Literaturhinweisen (auch zur in der Landessprache abgefassten Literatur) siehe Günter Trautmann, Fraktionen in Italien: ein Instrument der Parteien, in: Ludger Helms (Hrsg.), a.a.O. (Fn. 31), S. $121-144$.

66 Siehe Luca Verzichelli / Maurizio Cotta, Italien: Von ,beschränkten' Koalitionen zu alternierenden Regierungen?, in: Wolfgang C. Müller / Kaare Strøm (Hrsg.), Koalitionsregierungen in Westeuropa, Wien 1997, S. 547 - 626; Carol Mershon, The Cost of Coalition: A Five-Nation Comparison, in: Shaun Bowler / David M. Farrell / Richard S. Katz (Hrsg.), a.a.O. (Fn. 14), S. 227 - 268.

67 Siehe Giuseppe DiPalma, Surviving without Governing: the Italian Parties in Parliament, Berkeley 1977.

68 Siehe David Hine, Governing Italy. The Politics of Bargained Pluralism, Oxford 1993; Maurizio Cotta, The Rise and Fall of the ,Centrality' of the Italian Parliament: Transformations of the Executive-Legislative Subsystem after the Second World War, in: Gary W. Copeland / Samuel C. Patterson (Hrsg.), Parliaments in the Modern World. Changing Institutions, Ann Arbor 1994, S. 59 - 84. Dass die Diskussion um die „Heckenschützen“ auch ihren Niederschlag in der Debatte um die Parlamentsreform gefunden hat, zeigt Moritz Müller-Wirth, Die Debatte um die Parlamentsreform in Italien von 1971 - 1988, Frankfurt am Main 1992.

69 Siehe Massimiliano Landi / Riccardo Pelizzo, A Spatial Analysis of the XIII Italian Legislature, Singapore Management University, SMU Economics and Statistics Working Paper Series, Paper No. 22/2005. 
Newell befasste sich ausdrücklich mit Fraktionsgeschlossenheit und verglich diese mit der Zeit der Ersten Republik. ${ }^{70}$ Federico Ferrara wertete einen Datensatz von mehr als fünftausend elektronischen Voten aus der Zeit von 2001 bis 2002 aus. Er versuchte, die Abweichungsneigung der Abgeordneten in Abhängigkeit von ihrem im Mischwahlsystem beschrittenen elektoralen Pfad zu erklären. ${ }^{71}$ Weitere Studien befassten sich mit der Stabilität von Wahlkoalitionen und den Effekten des Mischwahlsystems, mit der Leistungsfähigkeit italienischer Regierungen und den Problemen der Konsolidierung des neuen Parteiensystems sowie mit den innerparteilichen Faktionen. ${ }^{72}$

Für das übrige Westeuropa findet sich Literatur zu folgenden Fällen: Das hohe Ausmaß von Fraktionsgeschlossenheit in Österreich wurde im Rahmen einer umfassenden Abgeordnetenstudie behandelt. ${ }^{73}$ Von der Geschlossenheit der Fraktionen im spanischen Congreso vermittelte Sanchez de Dios einen ersten Eindruck. ${ }^{74}$ Für die Schweiz mit ihrem speziellen Regierungssystem lieferten Prisca Lanfranchi und Ruth Lüthi Daten. ${ }^{75}$ Im Übrigen finden sich einige hilfreiche Anmerkungen zur Geschlossenheit bezüglich einer Reihe genannter und nicht genannter westeuropäischer Parlamente in einschlägigen Sammelbänden. ${ }^{76}$

70 Siehe James L. Newell, Turning Over a New Leaf? Cohesion and Discipline in the Italian Parliament, in: Journal of Legislative Studies, 6. Jg. (2000), H. 1, S. $29-52$.

71 Siehe Federico Ferrara, a.a.O. (Fn. 26).

72 Siehe Aldo Di Virgilio, Electoral Alliances: Party Identities and Coalition Games, in: European Journal of Political Research, 34. Jg. (1998), H. 5, S. 5 - 33; Giliberto Capano / Marco Giuliani, Governing Without Surviving? An Italian Paradox: Law-Making in Italy, 1987 - 2001, in: Journal of Legislative Studies, 7. Jg. (2001), H. 4, S. 13 - 36; Luca Verzichelli, Much Ado about Something? Parliamentary Politics in Italy Amid the Rhetoric of Majority Rule and an Uncertain Party System, in: Journal of Legislative Studies, 9. Jg. (2003), H. 2, S. 35 - 55; Daniela Giannetti / Michael Laver, Party Cohesion, Party Discipline, Party Factions in Italy, in: Daniela Giannetti (Hrsg.), Intra-party Politics and Coalition Governments, London 2009. Zur Einschätzung der Fraktionsgeschlossenheit sind ferner die Muster der Parteiwechsel hilfreich, siehe William B. Heller / Carol Mershon, Party Switching in the Italian Chamber of Deputies, 1996 - 2001, in: Journal of Politics, 67. Jg. (2005), H. 2, S. 536 - 559. Zu Parteiwechseln in Italien (und Russland) siehe zudem Carol Mershon / Olga Shvetsova, Parliamentary Cycles and Party Switching in Legislatures, in: Comparative Political Studies, 41. Jg. (2008), H. 1, S. 99 - 127.

73 Siehe Wolfgang C. Müller u.a., a.a.O. (Fn. 9); siehe auch ders. / Barbara Steininger, Not yet the Locus of Power: Parliamentary Party Groups in Austria, in: Knut Heidar / Rund Koole (Hrsg.), a.a.O. (Fn. 31), S. $71-88$.

74 Siehe Sanchez de Dios, Parliamentary Party Discipline in Spain, in: Shaun Bowler / David M. Farrell / Richard S. Katz (Hrsg.), a.a.O. (Fn. 14), S. 141 - 166. Vgl. auch Sanchez de Dios, Output of the Spanish Cortes, in: European Journal of Political Research, 45. Jg. (2006), H. 4, S. 551 - 579; Lynn M. Maurer, Parliamentary Influence in a New Democracy: The Spanish Congress, in: Journal of Legislative Studies, 5. Jg. (1999), H. 2, S. $24-45$.

75 Siehe Prisca Lanfranchi / Ruth Lüthi, a.a.O. (Fn. 17) (zum Schweizer Nationalrat auch Ruth Lüthi, Die Legislativkommissionen der Schweizerischen Bundesversammlung: institutionelle Veränderungen und das Verhalten von Parlamentsmitgliedern, Wien 1997).

76 Siehe vor allem Lawrence D. Longley / Reuven Y. Hazan, On the Uneasy, Delicate, yet Necessary Relationships between Parliamentary Members and Leaders, in: Journal of Legislative Studies, 5. Jg. (1999), H. 3, S. 1 - 22. Siehe auch Wolfgang C. Müller / Thomas Saalfeld (Hrsg.), Members of Parliament in Western Europe: Roles and Behavior, London 1997; Wolfgang C. Müller / Kaare Strøm (Hrsg.), Koalitionsregierungen in Westeuropa: Bildung, Arbeitsweise und Beendigung, Wien 1997; Knut Heidar / Rund Koole, a.a.O. (Fn. 31); Ludger Helms (Hrsg.), a.a.O. (Fn. 31); Kaare Strøm / Wolfgang C. Müller / Torbjörn Bergman (Hrsg.), Delegation and Accountability in European Integration: the Nordic Parliamentary Democracies and the European Union, London 2000. 
Für Skandinavien ist insbesondere die Studie von Torben K. Jensen mit der Darstellung zu Mustern innerfraktioneller Geschlossenheit in Dänemark, Finnland, Island, Norwegen und Schweden von Bedeutung. Sein Beitrag fußte auf einer Vielzahl von Studien, die oft in den jeweiligen Landessprachen abgefasst sind und insgesamt reichhaltiges Datenmaterial bereitstellen. Die Fraktionsgeschlossenheit wurde für nahezu jedes der betrachteten Länder im Zeitraum der 90er Jahre sowie auch für einen gewissen Zeitraum davor untersucht. So können Veränderungen innerhalb eines Parlaments sichtbar werden. Der Beitrag enthält zudem Kausalzusammenhänge aufspürende Analysen in Form eines Vergleichs ähnlicher Fälle. ${ }^{77}$ Weiterhin finden sich zwei von Asbjørn Skjeveland verfasste Studien zum dänischen Folketing, die in anschaulicher Weise das Phänomen innerfraktioneller Geschlossenheit vor Augen führen. ${ }^{78}$ Daten für Finnland legte zudem Lauri Karvonen ${ }^{79}$, für Norwegen Bjørn Erik Rasch vor. ${ }^{80}$

Gegenstand recht intensiver Forschungen ist auch das EP - aus ähnlichen Gründen wie bei der Russischen Staatsduma (siehe unten). Noch mehr als diese eignet sich das EP nämlich aufgrund seiner heterogenen Zusammensetzung als „Laboratorium des Politikwissenschaftlers“, etwa auch um das Verhältnis von Disziplin und Kohäsion zu bestimmen. ${ }^{81}$ Tapio Raunio beobachtete recht große Geschlossenheit, die - wie Simon Hix, Abdul Noury und Gérard Roland nachwiesen - im Verlauf der Jahre zugenommen hat. ${ }^{82}$ Shaun Bowler und David M. Farrell stießen in ihrer Abgeordnetenbefragung zur Fraktionsgeschlossenheit allerdings auf sehr unterschiedliche Einstellungen der Parlamentarier. ${ }^{83}$ Simon Hix analysierte die Selektion der Kandidaten und wahlsystemspezifische Einflüsse auf das Verhalten der Abgeordneten. ${ }^{84}$ Janina Thiem vermutete in ihrer Studie zum Einfluss der nationalen Parteien auf das Verhalten der MdEP mit - auch für den internationalen Vergleich - erwä-

77 Siehe Torben K. Jensen, a.a.O. (Fn. 12).

78 Siehe Asbjørn Skjeveland, a.a.O. (Fn. 12); ders., Party Cohesion in the Danish Parliament, in: Journal of Legislative Studies, 7. Jg. (2001), H. 2, S. 35 - 56.

79 Siehe Lauri Karvonen, Preferential Voting: Incidence and Effects, in: International Political Science Review, 25. Jg. (2004), H. 2, S. 203 - 226, S. 219. Zur Fraktionsgeschlossenheit im finnischen Abgeordnetenhaus des Jahres 2003 siehe Antti Pajala / Aleks Jakulin / Buntine Wray, Parliamentary Party Group and Individual Voting Behavior in the Finnish Parliament in Year 2003: A Group Cohesion in Voting Similarity Analysis, Working Paper, University of Turku 2005 (verfügbar unter: http://vanha.soc.utu.fi/valtio-oppi/mopi/misc/pajala_jakulin_buntine_vers_1. pdf, Abruf am 1. August 2009). Zu Finnland (und Schweden) siehe auch die einschlägigen Beiträge in Knut Heidar / Rund Koole, a.a.O. (Fn. 31).

80 Siehe Bjørn Erik Rasch, a.a.O. (Fn. 24); siehe auch die Nachweise bei Hanne Marthe Narud I Kaare Strøm, Norway: Madisonianism Reborn, in: Scandinavian Political Studies, 27. Jg. (2004), H. 2, S. $175-201$, S. 189.

81 Vgl. Thorsten Faas, a.a.O. (Fn. 26), S. 849.

82 Siehe Tapio Raunio, The Challenge of Diversity: Party Cohesion in the European Parliament, in: Shaun Bowler / David M. Farrell / Richard S. Katz (Hrsg.), a.a.O. (Fn. 14), S. 189 - 207; Simon Hix / Abdul Noury / Gérard Roland, Power to the Parties: Cohesion and Competition in the European Parliament, 1979-2001, in: British Journal of Political Science, 35. Jg. (2005), H. 2, S. $209-234$.

83 Siehe Shaun Bowler / David M. Farrell, Parties and Party Discipline within the European Parliament: A Norms-Based Approach, in: dies. / Richard S. Katz (Hrsg.), a.a.O. (Fn. 14), S. 208 222.

84 Siehe Simon Hix, a.a.O. (Fn. 26). Ähnlich wie dieser geht Thorsten Faas, a.a.O. (Fn. 26), vor, der zudem das Verhältnis der Abgeordneten zu ihren Parteien in den Blick nimmt. 
genswerten Argumenten, dass das Ausmaß der Geschlossenheit systematisch überschätzt werde. Dies sei der Fall, weil Fraktionsführer die namentlichen Abstimmungen in strategischer Absicht beantragten, nämlich um ihre Gruppe - erst im Fall großer Geschlossenheit (!) - am Wählermarkt zu positionieren. ${ }^{85}$ Aufgrund der guten Datenlage kommen bei den Forschungen zum EP viele jener statistischen Modelle zum Einsatz, die bei den Analysen der US-Parlamente entwickelt wurden. ${ }^{86}$

\section{Parlamente in Ost- und Mitteleuropa}

In Ost- und Mitteleuropa hat vor allem die Staatsduma in Russland großes Interesse auf sich gezogen. ${ }^{87}$ Die Parteienlandschaft ist sehr heterogen, bis 2004 bestand ein Mischwahlsystem, und die große Anzahl namentlicher Abstimmungen macht statistische Analysen leicht möglich. Neben dem EP ist die Duma also ein ideales „politikwissenschaftliches Laboratorium“. Entsprechend gingen Moshe Haspel, Thomas F. Remington und Steven S. Smith den verhaltensprägenden Effekten unterschiedlicher elektoraler Pfade zum Abgeordnetenmandat nach. ${ }^{88}$ Steven S. Smith und Thomas F. Remington hatten ähnliche Analysen in ihrer Monographie - auch mittels Surveydaten - durchgeführt. ${ }^{89}$ Weitere wahlsystemspezifische Effekte waren Gegenstand der neuesten Analysen von Jana Kunicová und Thomas F. Remington. ${ }^{90}$ Surveydaten verarbeiteten auch Herbert Kitschelt und Regina Smyth, deren Studie die programmatische Kohäsion der Parteien unter anderem in Russland erfasste. ${ }^{91}$ Paul Chaisty sammelte Daten zu den Mustern von Parteiwechseln und zur Abstimmungsgeschlossenheit. ${ }^{92}$ Thomas F. Remington untersuchte die Muster der Unterstützung des Präsidenten vor und nach Wladimir Putins Amtsübernahme ${ }^{93}$, und Frank C. Thames befasste

85 Janina Thiem, a.a.O. (Fn. 15). Zu weiteren Kausalanalysen siehe Simon Hix, Parliamentary Behaviour with Two Principals: Preferences, Parties and Voting in the European Parliament, in: American Journal of Political Science, 46. Jg. (2002), H. 3, S. 688 - 698; Abdul Noury, Ideology, Nationality and Euro-Parliamentarians, in: European Union Politics, 3. Jg. (2002), H. 1, S. 33 - 58; siehe auch den Literaturbericht bei Janina Thiem, a.a.O. (Fn. 15), S. 29 ff.

86 Die Forschungen sind zusammengefasst bei Simon Hix / Abdul Noury / Gérard Roland, Democratic Politics in the European Parliament, Cambridge 2007, insbesondere S. 87 ff. Bei diesen Autoren finden sich Daten zu allen Wahlperioden bis 2004. Unter http://personal.lse.ac.uk/hix/ stehen diese Daten für Analysen zur Verfügung.

87 Einen sehr guten Einstieg in die russische Parlamentsentwicklung nach 1994 liefert Thomas F. Remington, The Russian Federal Assembly, 1994 - 2004, in: Journal of Legislative Studies, 13. Jg. (2007), H. 1, S. $121-141$.

88 Siehe Moshe Haspel / Thomas F. Remington / Steven S. Smith, a.a.O. (Fn. 26). Zur Beantwortung einer ähnlichen Fragestellung führt Frank C. Thames einen Vergleich der Duma, des ukrainischen und des ungarischen Parlaments durch, a.a.O. (Fn. 26).

89 Siehe Steven S. Smith / Thomas F. Remington, a.a.O. (Fn. 26), insbesondere S. 116 - 136.

90 Siehe Thomas F. Remington und Jana Kunicová, a.a.O. (Fn. 26).

91 Siehe Herbert Kitschelt / Regina Smyth, Programmatic Party Cohesion in Emerging Postcommunist Democracies: Russia in Comparative Context, in: Comparative Political Studies, 35. Jg. (2002), H. 10, S. 1228 - 1256. Die Autoren betrachten neben Russland auch Tschechien, Bulgarien, Ungarn und Polen.

92 Siehe Paul Chaisty, Party Cohesion and Policy-Making in Russia, in: Party Politics, 11. Jg. (2005), H. 3, S. 299 - 318. Zu Parteiwechseln in Russland (und Italien) siehe zudem Carol Mershon / Olga Shvetsova, a.a.O. (Fn. 72).

93 Siehe Thomas F. Remington, a.a.O. (Fn. 21). 
sich außerdem mit den von der Konsolidierung der Parteiensysteme ausgehenden Effekten auf die Fraktionsgeschlossenheit. ${ }^{94}$ Auch in Zukunft wird die Duma wegen der systemtransformativen Phase, in der sich das heutige Russland befindet, ein für die Forschung außerordentlich spannendes Parlament darstellen.

Für das übrige Osteuropa, das reichlich Gelegenheit bietet, sehr „unruhige“ Fälle zu untersuchen, gibt es nur wenige Studien und Befunde. Attila Ágh zeichnete die Entwicklung der Fraktionsgeschlossenheit in Ungarn nach. ${ }^{95}$ Für Tschechien gibt es Untersuchungen von Petr Kopecký und - zur Organisation und Stellung dortiger Fraktionen - von Lukáš Linek und Petra Rakušanová. ${ }^{96}$ Daten zur Abstimmungsgeschlossenheit finden sich bei Frank C. Thames für die Ukraine, bei Alvidas Lukošaitis für Litauen und bei Maja Sever und Tomaz Dezelan für Slowenien. ${ }^{97}$ Kuba Zielinski und Natalie Kistner präsentierten Daten zu Polen. ${ }^{98}$

94 Siehe Frank C. Thames, a.a.O. (Fn. 46).

95 Siehe Attila Agh, The Parliamentarization of the East Central European Parties: Party Discipline in the Hungarian Parliament, 1990 - 1996, in: Shaun Bowler / David M. Farrell / Richard S. Katz (Hrsg.), a.a.O. (Fn. 14), S. 167 - 188. Außerdem ist Ungarn in der vergleichenden Untersuchung von Frank C. Thames, a.a.O. (Fn. 26), einbezogen, und in naher Zukunft wird folgender Beitrag mit Daten zur Fraktionsgeschlossenheit (Jahre 2000, 2003 und 2005) vorliegen: Gabriella Ilonszki / Krisztina Jáger, Eros kormány - gyenge parlament? A törvényhozási kapacitás és törvényhozási teljesítmény politikai szociológiai összefüggései, in: Századvég, im Erscheinen.

96 Siehe Petr Kopecký, The Limits of Whips and Watchdogs: Parliamentary Parties in the Czech Republic, in: Knut Heidar / Ruud Koole (Hrsg.), a.a.O. (Fn. 31), S. 177 - 194; Lukáś Linek / Petra Rakuśanová, Why Czech Parliamentary Party Groups Vote Less Unitedly. The Role of Frequent Voting and Big Majorities in Passing Bills, in: Sociologický casopis / Czech Sociological Review, 41. Jg. (2005), H. 3, S. 423 - 442; ausführlich Lukás Linek / Petra Rakušanová, Parties in the Parliament. Why, When and How Do Parties Act in Unity? Parliamentary Party Groups in the Chamber of Deputies in the Years 1998 - 2002, Prague Institute of Sociology, Academy of Sciences of the Czech Republic 2002. Siehe zum tschechischen Abgeordnetenhaus ferner Abdul Noury / Elena Mielcova, Electoral Performance and Voting Behavior: Evidence from the Czech Parliament 1992 - 2002, University of California, Institute of Governmental Studies, WP 200514, Berkeley 2005 (verfügbar unter http://repositories.cdlib.org/igs/WP2005-14, Abruf am 1. August 2009).

97 Siehe Frank C. Thames, a.a.O. (Fn. 46); ders., a.a.O. (Fn. 26). Von ihm wurden zudem die Muster der Parteiwechsel in der Ukraine untersucht: Frank C. Thames, Searching for the Electoral Connection: Parliamentary Party Switching in the Ukrainian Rada, 1998 - 2002, in: Legislative Studies Quarterly, 32. Jg. (2007), H. 2, S. 223 - 256; Alvidas Lukošaitis, Lietuvos Parlamento Frakciju Vienybè ir Drausmè, in: Politologija, 33. Jg. (2004), H. 1, S. 1 - 40 (zu Litauen siehe auch: Terry D. Clark / Žilvinas Martinaitis / Ramünas Dilba, a.a.O. (Fn. 26)); Maja Sever / Tomaz Dezelan, Deputy Group Cohesion in Parliamentary Roll-Call Voting in Slovenia, in: Central European Political Science Review, 5. Jg. (2004), H. 3, S. 65 - 83.

98 Siehe Kuba Zielinski, Party Cohesion in the Polish Sejm: An Exploration, Paper presented at the 2004 Annual Meeting of the American Political Science Association; Natalie Kistner, Party Discipline and Legislative Institutionalization in Poland, Paper presented at the 2006 Midwest Political Science Association Conference, Chicago 2006. Zu den einschlägigen Problemen geringer Fraktionsgeschlossenheit in Polen siehe Radoslaw Zubek, Parties, rules and government legislative control in Central Europe: The case of Poland, in: Communist and Post-Communist Studies, 41. Jg. (2008), H. 2, S. 147 - 161. Polen ist zudem in der Vergleichsstudie von John M. Carey, a.a.O. (Fn. 13), erfasst. Eine vergleichende Arbeit zu Ungarn, Tschechien, der Slowakei und Polen wird von Gabriela Borz angefertigt. Zum Stand siehe dies., Determinants of Party Unity in Central Eastern Europe, in: CEU Political Science Review, 1. Jg. (2006), H. 3, S. 30 - 49. Für Osteuropa lohnt zudem der Blick in den Sammelband von Knut Heidar und Ruud Koole, a.a.O. (Fn. 31); dort finden sich Beiträge zu Ungarn, Tschechien und der Slowakei. 


\section{Parlamente in Lateinamerika (und der übrigen Welt)}

Für Lateinamerika liegen Studien zu einer ganzen Reihe von Parlamenten vor. ${ }^{99}$ Ein spezielles institutionelles Arrangement findet sich bei der mexikanischen Cámara Federal de Diputados: Deren Abgeordnete können nicht direkt für eine zweite Amtszeit wiedergewählt werden. Zu dieser Institution und der dortigen Fraktionsgeschlossenheit gibt es bereits ausführliche Analysen für die Zeit nach der Dominanz der Partido (PRI). Allen voran ist die Monographie von Laura Valencia Escamilla zu nennen. Sie nahm die Karrieremuster, die elektoralen Pfade, auf denen die Abgeordneten ins Parlament gelangen (es besteht ein Mischwahlsystem), die inneren Parteiorganisationen und das Verhältnis von Partei, Fraktion und Exekutive in den Blick. ${ }^{100}$ Effekte aufgrund verschiedener elektoraler Pfade sind auch Gegenstand des Beitrags von Jeffrey A. Weldon, und Benito Nacif lieferte eine knappe Übersicht über das „Modell der zentralisierten Parteien“ in Mexiko. ${ }^{101}$

Kontrovers ist die Forschung zur brasilianischen Câmara dos Deputados. Fernando Limongi und Argelina C. Figueiredo haben ein nennenswertes Niveau einheitlichen Abstimmens nachgewiesen, obwohl stets recht chaotisch organisierte Fraktionen vermutet wurden. ${ }^{102}$ Besonders geschlossen, fast schon auf dem Niveau westeuropäischer Fraktionen, agiert die Partido dos Trabalhadores. ${ }^{103}$ Kontrovers wird über die einschlägigen Kausalpro-

99 Siehe vor allem den Sammelband von Scott Morgenstern / Benito Nacif (Hrsg.), Legislative Politics in Latin America, Cambridge 2002. Siehe auch den Ansatz von Letecia Ruiz Rodríguez I Mercedes García Montero, Coherencia Partidista en las Élites Parlamentarias Latinoamericanas, in: Revista Espańola de Ciencia Política, 5. Jg. (2003), H. 8, S. 71 - 102.

100 Siehe Laura Valencia Escamilla, a.a.O. (Fn. 9).

101 Siehe Jeffrey A. Weldon, a.a.O. (Fn. 26); Benito Nacif, Understanding Party Discipline in the Mexican Chamber of Deputies: the Centralized Party Model, in: Scott Morgenstern / ders. (Hrsg.), a.a.O. (Fn. 99), S. 254 - 284. Weitere aktuelle Analysen stellen dar: Ángeles Mascott Sánchez, Disciplina Partidista en México: el Voto Dividido de las Fracciones Parlamentarias durante las LVII, LVIII y LIX Legislaturas. Centro de Estudio Sociales y de Opinión Pública. Documento de Trabajo número 1/2006 (verfügbar unter http://www3.diputados.gob.mx/camara/, Abruf am 20. Oktober 2008); Everardo Rodrigo Díaz Gómez, Desempeño Legislativo y Disciplina Partidista en Mexico: la Cámera de Diputados, 2000-2003, in: Confines de Relaciones Internacionales y Ciencia Política, 2. Jg. (2006), H. 3, S. 45 - 64; Jerónimo Díaz Rebolledo, Los determinantes de la Indisciplina Partidaria. Apuntes Sobre la Conexión Electoral en el Congreso mexicano, 2000-2003, in: Política y Gobierno, 12. Jg. (2005), H. 2, S. 313 - 330.

102 Siehe Fernando Limongi / Argelina C. Figueiredo, Partidos Políticos na Câmera dos Deputados: 1989 - 1994, in: Dados, 38. Jg. (1995), H. 3, S. 497 - 524; Argelina C. Figueiredo / Fernando Limongi, Executivo e Legislativo na Nova Ordem Constitucional, Rio de Janeiro 1999; dies., Presidential Power, Legislative Organization, and Party Behavior in Brazil, in: Comparative Politics, 32. Jg. (2000), H. 2, S. 151 - 170. Weitere Daten finden sich bei Jairo Nicolau, Disciplina Partidária e Base Parlamentar na Câmara dos Deputados no Primeiro Governo Fernando Henrique Cardoso (1995 - 1998), in: Dados, 43. Jg. (2000), H. 4, S. 709 - 734. Einen Einstieg in die Debatte liefert Octavio Amorim Neto, The Puzzle of Party Discipline in Brazil, in: Latin American Politics and Society, 44. Jg. (2002), H. 1, S. 127 - 144. Im internationalen Vergleich sind die Geschlossenheitswerte der Fraktionen der Câmera allerdings tatsächlich recht niedrig, und es lassen sich konstant hohe Parteiwechselraten beobachten, siehe Scott W. Desposato, Parties for Rent? Ambition, Ideology, and Party Switching in Brazil's Chamber of Deputies, in: American Journal of Political Science, 50. Jg. (2006), H. 1, S. 62 - 80.

103 Diese hohe Geschlossenheit zeigte die Fraktion auch vor der Präsidentschaft von Lula da Silva. Vgl. die in Fn. 102 angegebene Literatur. Siehe ferner Alan Daniel Freire De Lacerda, O PT e Unidade Partidária como Problema, in: Dados, 45. Jg. (2002), H. 1, S. 39 - 76. 
zesse hinter der geringen Geschlossenheit gestritten. ${ }^{104}$ Octavio Amorim Neto und Fabiano Santos haben sich zudem der Câmara dos Deputados von 1946 bis 1964 gewidmet - der Zeit also vor der Einrichtung der Militärdiktatur. Sie ermittelten dort besonders wenig Geschlossenheit. ${ }^{105}$

Für das übrige Lateinamerika sind noch folgende Studien zu erwähnen: Zu Argentiniens Cámara de Diputados liegt die umfassende und gestalterschließende Studie von Mark Jones vor. ${ }^{106}$ John M. Carey sowie Eduardo Alemán und Sebastián Saiegh haben für Chile, das ein einzigartiges Wahlrecht für sein Unterhaus besitzt, interessante Einsichten in die Kohäsion sowohl der Fraktionen als auch der Wahlkoalitionen festgehalten. ${ }^{107}$ Die vergleichende Untersuchung von Scott Morgenstern schloss neben Argentinien, Brasilien und Chile Uruguay ein. ${ }^{108}$ John M. Carey lieferte außerdem Daten zu Ecuador, Guatemala, Nicaragua und Peru. ${ }^{109}$ Weitere Daten, auch qualitative Einschätzungen der Geschlossenheitsniveaus, stammen von Michael Coppedge. ${ }^{110}$

104 In dieser Hinsicht ist insbesondere der Beitrag von Barry Ames zu nennen, The Deadlock of Democracy in Brazil, Ann Arbor 2001; ders., Party Discipline in the Chamber of Deputies, in: Scott Morgenstern / Benito Nacif(Hrsg.), a.a.O. (Fn. 99), S. 185 - 221. Zur Koalitionsgeschlossenheit präsidentieller Koalitionen siehe Octavio Amorim Neto, Presidential Cabinets, Electoral Cycles, and Coalition Discipline in Brazil, in: Scott Morgenstern / Benito Nacif (Hrsg.), a.a.O. (Fn. 99), S. 48 - 78; John M. Carey / Gina Yannitell Reinhardt, State-Level Institutional Effects on Legislative Coalition Unity in Brazil, in: Legislative Studies Quarterly, 29. Jg. (2004), H. 6, S. 23 - 47 (zum dort gleichfalls betrachteten Effekt des brasilianischen Föderalismus auf die Geschlossenheit siehe auch David Samuels, Progressive Ambition, Federalism, and Pork-Barreling in Brazil, in: Scott Morgenstern / Benito Nacif(Hrsg.), a.a.O. (Fn. 99), S. 315 - 340; ders., Ambition, Federalism, and Legislative Politics in Brazil, Cambridge 2003; Scott W. Desposato, The Impact of Federalism on National Party Cohesion in Brazil, in: Legislative Studies Quarterly, 29. Jg. (2004), H. 2, S. 259 - 285). Zu den Regeln in der Kammer siehe Fabiano Santos I Lucio Rennó, The Selection of Committee Leadership in the Brazilian Chamber of Deputies, in: Journal of Legislative Studies, 10. Jg. (2004), H. 1, S. 50 - 70.

105 Siehe Octavio Amorim Neto / Fabiano Santos, The Executive Connection: Presidentially Defined Factions and Party Discipline in Brazil, in: Party Politics, 7. Jg. (2001), H. 2, S. 213 - 234. Befunde zu einem weiteren brasilianischen ,historischen Fall”, dem Verfassungsgebenden Kongress von 1988, liefern: Scott P. Mainwaring / Aníbal Pérez Liñán, Party Discipline in the Brazilian Constitutional Congress, in: Legislative Studies Quarterly, 22. Jg. (1997), H. 4, S. 453 - 483; Scott Mainwaring, Rethinking Party System in the Third Wave of Democratization: The Case of Brazil, Stanford 1999.

106 Siehe Mark P. Jones, Explaining the High Levels of Party Discipline in the Argentine Congress, in: Scott Morgenstern / Benito Nacif (Hrsg.), a.a.O. (Fn. 99), S. 147 - 184. Dem Einfluss regionaler Faktoren und regionaler Parteiführer auf den politischen Prozess des Gesamtsystems sowie auf die Geschlossenheit widmen sich Mark P. Jones / Wonjae Hwang, Party Government in Presidential Democracies: Extending Cartel Theory Beyond the U.S. Congress, in: American Journal of Political Science, 49. Jg. (2005), H. 2, S. 267 - 282.

107 Siehe John M. Carey, Parties, Coalitions, and the Chilean Congress in the 1990s, in: Scott Morgenstern / Benito Nacif(Hrsg.), a.a.O. (Fn. 99), S. 222 - 253; Eduardo Alemán / Sebastián Saiegh, Legislative Preferences, Political Parties, and Coalition Unity in Chile, in: Comparative Politics, 39. Jg. (2007), H. 3, S. $253-272$.

108 Siehe Scott Morgenstern, a.a.O. (Fn. 33).

109 Siehe John M. Carey, a.a.O. (Fn. 13); ders., a.a.O. (Fn. 39).

110 Siehe Michael Coppedge, The Dynamic Diversity of Latin American Party Systems, in: Party Politics, 4. Jg. (1998), H. 4, S. 547 - 568. Sowohl dessen als auch die Daten von John M. Carey finden sich in dem derzeit wohl umfänglichsten Datensatz zur Fraktionsgeschlossenheit von Steffen Kailitz, a.a.O. (Fn. 1). 
Für die übrige Welt sind Daten für Neuseeland und die Philippinen verfügbar. ${ }^{111}$ Jonathan Malloy legte die Strukturen der Fraktionsgeschlossenheit samt der interessanten Kombination aus relativ offenem Nominierungsverfahren und geringer Parteibindung in Kanada dar. ${ }^{12}$ Gideon Rahat untersuchte die Entwicklung der Fraktionsgeschlossenheit in Israel. ${ }^{113}$ Eine Einzelfallstudie zum Abstimmungsverhalten der Liberalen Demokratischen Partei Japans (LDP) bei der Abstimmung zur Privatisierung der japanischen Post erstellten Kunikai Nemoto, Ellis Krauss und Robert Pekkanen. ${ }^{114}$

\section{Ergebnisse, Defizite und erforderliche Anstrengungen der Forschung}

Das globale Panorama der unterschiedlichen Geschlossenheit von Parlamentsfraktionen lässt sich zwar schon erahnen, allerdings fehlt für einige Weltregionen wünschenswertes Datenmaterial. Hier ist grundlegende, mit langem Atem betriebene empirische Forschung in den Archiven vieler Parlamente nötig. Dabei sollten auch Zeitreihen ermittelt werden, um Veränderungsprozesse zu erfassen. Eine Bereicherung stellte dar, wenn entsprechende Daten nicht nur für die parlamentarische Praxis in Gegenwart und jüngerer Vergangenheit, sondern auch für historische Parlamente beschafft würden. Sie hülfen nämlich bei der Erkennung von Mustern der Genese von Fraktionen und Parteien. Die Analyse nicht mehr existierender Parlamente steigerte zudem die Vielfalt betrachteter institutioneller Konfigurationen, was wiederum die vergleichende Kausalanalyse bereichern könnte. Bei der Beschaffung einschlägiger Informationen zur Geschlossenheit ist generell zu erwägen, welche hilfreichen Kriterien sich finden ließen, um die Geschlossenheit von Fraktionen einzuschätzen, wenn namentliche Abstimmungen nicht verfügbar sind. ${ }^{115}$

Hinsichtlich der Kausalprozesse hinter den unterschiedlichen Geschlossenheitsniveaus kann als gesichert gelten, dass Fraktionen in parlamentarischen Regierungssystemen grundsätzlich geschlossener agieren als jene im Präsidentialismus, wenn auch letztere keineswegs ohne Geschlossenheit handeln. Dies liegt vor allem an der regierungstragenden Funktion der Mehrheitsparteien und der von oppositionellen Fraktionen zu erfüllenden Alternativfunktion. Daneben sind innerfraktionelle Arbeitsteilung und - oft in Einzelfallstudien anschaulich dargestellt - ideologisch-weltanschauliche Kohärenz, Gruppenbewusstsein und soziale Identität Ursachen des Mit- und Füreinanders in den Fraktionen. Außerdem wird dem Wahlsystem und der Kandidatenselektion weithin ein nicht unwichtiger Einfluss zu-

111 Siehe John M. Carey, a.a.O. (Fn. 13).

112 Siehe Jonathan Malloy, High Discipline, Low Cohesion? The Uncertain Patterns of Canadian Parliamentary Party Groups, in: Journal of Legislative Studies, 9. Jg. (2003), H. 4, S. 116 129.

113 Siehe Gideon Rahat, Determinants of Party Cohesion: Evidence from the Case of the Israeli Parliament, in: Parliamentary Affairs, 60. Jg. (2007), H. 2, S. 279 - 296.

114 Siehe Kuniaki Nemoto / Ellis Krauss / Robert Pekkanen, Policy Dissension and Party Discipline: the July 2005 Vote on Postal Privatization in Japan, in: British Journal of Political Science, 38. Jg. (2008), H. 3, S. $499-525$.

115 Vgl. entsprechende Überlegungen bei Kenneth Janda, a.a.O. (Fn. 40), S. 119; Michael Coppedge, a.a.O. (Fn. 110); Steffen Kailitz, a.a.O. (Fn. 1); Erik Fritzsche, Innerfraktionelle Geschlossenheit im internationalen Vergleich: Eine qualitative Vergleichsanalyse zwischen holistischem Einzelfallverständnis und variablenorientierter Kausalanalyse, Magisterarbeit, TU Dresden 2008 (verfügbar unter www.erik-fritzsche.de/fraktionsgeschlossenheit, Abruf am 1. August 2009). 
gesprochen, wobei allerdings unklar bleibt, inwieweit diese separierten Faktoren einander durchdringen, welche Wirkung stärker ist und welche weiteren Kontexte die Funktionslogik womöglich entscheidend mitprägen. Das Wahlsystem allein scheint dagegen wenig effektvoll: Die Studien an Mischwahlsystemen zeigen auf, dass die beliebte Hypothese vom egoistischen Wahlkreisabgeordneten, der dem linientreuen Listenabgeordneten entgegenzusetzen sei, keinesfalls der Wirklichkeit entspricht. ${ }^{116}$

Mit dem dominanten Effekt des Regierungssystems, der weithin bekannt ist beziehungsweise einfach unterstellt wird, ist für viele das Phänomen der Fraktionsgeschlossenheit hinreichend abgehandelt. Doch ein näherer Blick auf das Phänomen zeigt Erklärungsbedürftiges: Fraktionen in präsidentiellen Regierungssystemen haben ganz unterschiedliche Geschlossenheitsniveaus; einige Fraktionen in parlamentarischen Regierungssystemen agieren mit relativ wenig Geschlossenheit (mit entsprechenden Folgen für die Stabilität der Regierung und oft auch des gesamten politischen Systems). Man darf zudem nicht außer Acht lassen, dass ganz unterschiedliche Ursachen für sehr ähnliche Geschlossenheitsniveaus verantwortlich sein können. Neben der Wichtigkeit einheitlichen Agierens für die Regierungsstabilität und für den Wettbewerb um Wählerzuspruch sind auch andere Mechanismen der Herstellung von Fraktionsgeschlossenheit denkbar, und zwar auch solche, die in populären delegitimierenden Vermutungen zum Abgeordnetenverhalten durchaus Anklang finden (vor allem eine angeblich von Parteiführern betriebene Ausgrenzung jener Abgeordneten, die sich der Fraktionslinie verweigern). Auch „Aufklärer“ dürften sich also von der - vergleichend durchgeführten - Kausalanalyse des Phänomens interessante Einsichten darüber erhoffen, wie stichhaltig die abfälligen Vermutungen wirklich sind.

Solche Anstrengungen würden gewiss auch von einigen theoretischen Erweiterungen unserer vorherrschenden Modelle profitieren, die mehr als bisher dem hier als Kohäsion bezeichneten Sockel innerfraktioneller Geschlossenheit Rechnung tragen sollten. ${ }^{17}$ Das theoretische Hantieren mit rationalen Kalkülen allein mutet dabei etwas eigentümlich an. Es erscheint doch eigentlich sehr erklärungsbedürftig, warum sich Menschen in relativ kleinen Gruppen zusammenfinden sollten, wie sie dies in Fraktionen gewöhnlich tun, nur um dann nicht halbwegs geschlossen zu agieren. Fraktions- und Gruppengeschlossenheit scheitert eher dort, wo nicht nur disziplinierende Faktoren, sondern zudem auch zur Kohäsion führende Ursachen fehlen. Solche Kohäsionseffekte können eruiert werden, wenn folgende Dinge in den Blick genommen würden: die Rekrutierung und Sozialisation von Kandidatenanwärtern; die Kandidatenselektion; Geschichte und Ideologie sowie die inhaltliche, weltanschauliche, biographische und generationelle Homogenität der Parteien und Fraktionen ${ }^{118}$; die Erwartungen der Bürger in Bezug auf die Geschlossenheit der politischen Gruppen; und der „Markenwert“ von Parteien im politischen Wettbewerb.

116 Selbst die Berücksichtigung einiger intervenierender Variablen (etwa die Konkurrenz im Wahlkreis) führt nur zur Beobachtung geringer Effekte. Siehe die in Fn. 26 angegebene Literatur zu den jeweiligen Einzelbefunden. Der für Deutschland einschlägige, sehr eindrucksvolle Befund findet sich bei Michael Becher / Ulrich Sieberer, a.a.O. (Fn. 26).

117 Dieser „Sockel“ wird beim Blick auf das Panorama der vorfindbaren Fraktionsgeschlossenheit ziemlich gut deutlich, vgl. etwa Steffen Kailitz, a.a.O. (Fn. 1).

118 Beispielsweise lässt sich die zunehmende Geschlossenheit der CDU/CSU-Bundestagsfraktion in den 1950er Jahren als eine zunehmende Homogenisierung von Partei und Fraktion deuten, siehe Thomas Saalfeld, a.a.O. (Fn. 6). Ähnliches scheint für viele Fraktionen in den postsozialistischen Parlamenten Mittel- und Osteuropas für die Zeit nach deren Gründung zu gelten. 
Daten zu solchen Einflussgrößen zu erheben und vergleichend zu analysieren ist unbedingt notwendig: Nur solche Studien eignen sich zur produktiven Theoriebildung. Allerdings liegt darin eine große Herausforderung. Es wundert daher nicht, dass sie auch im vorliegenden Forschungsfeld bisher äußerst spärlich vertreten sind. Vergleichsbehindernd wirkt sich aus, dass - erstens - idiographische Einzelfallstudien zu Parlamenten relativ selten sind: Das trifft besonders für Parlamente in Asien und Afrika, oft auch für jene in Lateinamerika und Osteuropa sowie für historische Parlamente zu. Gerade zu den kausalanalytisch einschlägigen Strukturen des Parlamentarismus - beispielsweise wie Abgeordnete ihre Rolle sehen und konkret ausfüllen, welchen Rekrutierungs- und Selektionsprozessen sie sich stellen oder wie sie sozialisiert werden - finden sich nur sehr selten für den Vergleich nützliche Informationen. Hierfür ist nicht zuletzt verantwortlich, dass vergleichende Forschungsprojekte - das zeigt sich auch in den Arbeiten zur Fraktionsgeschlossenheit - viel lieber auf Korrelationsanalysen verfügbarer intervallskalierter Daten gestützt werden statt auf qualitatives Material, etwa Interviews. ${ }^{119}$

Ein zweiter vergleichserschwerender Umstand liegt in den dominanten Datenanalysestrategien der vorfindbaren Vergleichsdesigns: Die statistischen Verfahren des empirischen Tests von Hypothesen verlangen oft vergleichbare Daten auf hohem Datenniveau und in jedem Fall ein günstiges Verhältnis von Fallzahl und Varianz. Schon für die abhängige Variable entstehen hier allerdings große Probleme: Die Analyse innerfraktioneller Geschlossenheit ist in vielen Parlamenten durch die schlichte Nichtverfügbarkeit von Daten zu namentlichen Abstimmungen drastisch eingeschränkt, da diese selten oder gar nicht stattfinden. Zudem werden die dafür üblichen Maßzahlen zur Verdichtung des Grades gemeinsamen Abstimmens zwar immer wieder in ihrer Basis recht ähnlich, im Detail dann aber durchaus verschieden berechnet. ${ }^{120}$ Dies führt zu Schwierigkeiten beim Vergleich sol-

119 Im vorliegenden Forschungskontext etwa gewinnbringend eingesetzt sind - teils sehr strukturierte - Interviews etwa bei John M. Carey, a.a.O. (Fn. 39); Asbjørn Skjeveland, a.a.O. (Fn. 78); Sam Depauw, a.a.O. (Fn. 63); oder bei Werner J. Patzelt, a.a.O. (Fn. 17). Weniger strukturiere Interviews ermöglichen aber vermutlich einen noch tieferen Einblick in die Kalküle, rollenprägenden Normen und handlungsleitenden Regeln der Abgeordneten, vgl. hierzu das Forschungsdesign von Werner J. Patzelt, Abgeordnete und ihr Beruf. Interviews, Umfragen, Analysen, Berlin 1995; vgl. auch die beiden Beiträge von Louise K. Davidson-Schmich, a.a.O. (Fn. 45).

120 Die Basis zur Messung der Fraktionsgeschlossenheit bildet üblicherweise der Rice-Index, bei dem der Anteil der Abweichler vom Anteil der zur Fraktionsmehrheit Zustimmenden abgezogen wird. Er besitzt einen Wertebereich von null (die Fraktion zerfällt in zwei hälftige Lager) bis eins (die Fraktionsmitglieder stimmen vollkommen gleich ab), siehe Stuart Rice, Quantitative Methods in Politics, New York 1928. Zumeist werden Enthaltungen als Neinstimmen gezählt. Absenzen werden entweder nicht berücksichtigt, mit einem Faktor gewichtet (so etwa Thomas Saalfeld, a.a.O. [Fn. 6]) oder bei Abstimmungen, in denen die absolute Mehrheit nötig ist, generell als Neinstimme gewertet (entsprechend müssen die Forscher etwa bei jeder Abstimmung in der Russischen Staatsduma vorgehen). Der Rice-Index ist nicht frei von Verzerrungen. Besonders problematisch ist, dass er nur bei namentlichen Abstimmungen verwendet werden kann, die jedoch keine Zufallsstichprobe darstellen und häufig besonders wichtige Abstimmungen beziehungsweise besonders einmütige Abstimmungen erfassen. Vgl. zur Problematik Janina Thiem, a.a.O. (Fn. 15); John M. Carey, a.a.O. (Fn. 13), S. 95 f.; Louise K. Davidson-Schmich, The Origins of Party Discipline, a.a.O. (Fn. 45); zur Möglichkeit einer Schätzung der Rice-Werte siehe Fn. 115. Weitere Probleme treten auf, wenn bestimmte Abstimmungen, etwa nach Bedeutung oder Knappheit, gewichtet werden. Für jeden Einzelfall mag dies durchaus dem Gegenstand sehr angemessen erscheinen, wirkt aber letztlich aufgrund der verschiedenen Operationalisierungen 
cher Werte. ${ }^{121}$ Hier wäre ein einheitlicher Standard, der in den Forschungspublikation zur Messung der Geschlossenheit in jedem Fall auch - nicht bloß (!) - verwendet würde, sehr wünschenswert.

Für die forschungslogischen Probleme mag es datenanalytische Instrumente geben, die eine gewisse Abhilfe schaffen, doch sind auch solche Innovationen keine Heilsbringer für Defizite in den Grundlagen der Parlamentarismusforschung. Datenprobleme sind nämlich oft nur Oberflächenphänomene von in der Tiefe liegenden theoretischen Schwierigkeiten. Daher scheint es, als könne gerade diesem Datenmangel, nebst der Lösung vieler hier relevanter Einzelfragen, nur dadurch begegnet werden, dass die Parlamentarismusforschung sich mehr als bisher auf ein integriertes Forschungsprogramm verständigt, das nachhaltig abgearbeitet wird. ${ }^{122}$ Dieses müsste von vornherein den theoriebildenden Vergleich zum Zweck haben und die Funktionsweise von Parlamenten (sowie deren Entwicklungsmuster) durch das Zusammenwirken von Einzelfall- und Vergleichsforschung erklären wollen. Dabei würden holistische Einzelfallstudien zu Parlamenten keineswegs abgewertet, sondern würden geradezu gefordert und ihre Datenliefer- und Interpretationshilfefunktion geschätzt (ohne sie hierauf reduzieren zu müssen). Das Fehlen solcher Beiträge dürfte für das Gros der heutigen Datenprobleme verantwortlich sein.

Ein solches Programm lässt dabei den individuellen Vorlieben der Forscher Raum. Es könnte durch seine der Forschung formgebende Struktur auch den Studierenden der Parlamentarismusforschung nützliche Orientierung bieten und durch die klare Benennung offener Fragen Forscherdrang entwickeln helfen. Von einem solchen integrierten Konzept ist die internationale Parlamentarismusforschung allerdings noch weit entfernt. Ihr ist deshalb eine wirklich kräftige Konjunktur zu wünschen - und zwar im ursprünglichen Wortsinn: ein alle verbindendes Vorhaben. Daraus könnte nicht zuletzt, aber auch ganz gewiss nicht als einzige, die Forschung am Phänomen der innerfraktionellen Geschlossenheit großen Nutzen ziehen.

vergleichserschwerend. Für kleine Parteien sind gesonderte Verzerrungseffekte zu beachten, siehe Scott W. Desposato, Correction for Small Group Inflation of Roll-Call Cohesion Scores, in: British Journal of Political Science, 35. Jg. (2005), H. 4, S. 731 - 744. Eine gute Einführung in die Funktionslogik der Indizes liefern Lee Anderson / Meredith W. Watts Jr. I Allen R. Wilcox, Legislative Roll-Call Analysis, Evanston 1966. Eine Einführung in den derzeitigen Stand räumlicher Modellierungen findet sich bei Keith T. Poole, a.a.O. (Fn. 34).

121 Bemühungen wie jene von John M. Carey mögen allerdings in gewissem Umfang Abhilfe schaffen. Er macht unter www.dartmouth.edu/ - jcarey/ (Abruf am 1. August 2009) seine umfassende Sammlung namentlicher Abstimmungen verfügbar und bietet obendrein Makros für Statistikprogramme an. Damit können bequem viele der möglichen Indizes (vgl. Fn. 120) zur Fraktionsgeschlossenheit berechnet werden.

122 Dass hier einiges im Argen liegt, ist keine neue Erkenntnis; vgl. etwa Suzanne S. Schüttemeyer, Vergleichende Parlamentarismusforschung, in: Dirk Berg-Schlosser / Ferdinand Müller Rommel (Hrsg.), Vergleichende Politikwissenschaft, Opladen 2003, S. 207 - 227; Werner J. Patzelt, a.a.O. (Fn. 6). Vgl. auch Werner J. Patzelt, Vergleichende Parlamentarismusforschung als Schlüssel zum Systemvergleich, in: Winfried Steffani / Uwe Thaysen (Hrsg.), Demokratie in Europa, Opladen 1995, S. 355 - 385; ders., Parlamentssoziologie, in: Viktoria Kaina / Andrea Römmele (Hrsg.), Politische Soziologie. Ein Studienhandbuch, Wiesbaden 2008, S. 311 - 351. 